quatrième série - tome 41 fascicule 6 novembre-décembre 2008

$$
\begin{aligned}
& \text { ANNALES } \\
& \text { SCIENTIFIQUES } \\
& \text { de } \\
& \text { L'ECOLE } \\
& \text { NORMALE } \\
& \text { SUPÉRIEURE }
\end{aligned}
$$

Jan Nekováŕ

Growth of Selmer groups of Hilbert modular forms over ring class fields 


\title{
GROWTH OF SELMER GROUPS OF HILBERT MODULAR FORMS OVER RING CLASS FIELDS
}

\author{
BY JAN NEKOVÁŘ
}

Abstract. - We prove non-trivial lower bounds for the growth of ranks of Selmer groups of Hilbert modular forms over ring class fields and over certain Kummer extensions, by establishing first a suitable parity result.

RÉsumÉ. - On donne des bornes inférieures non triviales sur la croissance des rangs des groupes de Selmer de formes modulaires de Hilbert sur les corps de classes d'anneau et sur des extensions de Kummer, en démontrant d'abord un résultat de parité.

\section{Introduction}

0.1. - Fix an algebraic closure $\overline{\mathbb{Q}}$ of $\mathbb{Q}$, a prime number $p$ and embeddings $i_{\infty}: \overline{\mathbb{Q}} \hookrightarrow \mathbb{C}$ and $i_{p}: \overline{\mathbb{Q}} \hookrightarrow \overline{\mathbb{Q}}_{p}$.

Let $F$ be a totally real number field and $g \in S_{k}(\mathfrak{n}, 1)$ a cuspidal Hilbert modular newform over $F$ of parallel weight $k$, trivial character (which implies that $k$ is even) and (exact) level $\mathfrak{n}$.

Let $K$ be a totally imaginary quadratic extension of $F$ and $\chi: \mathbb{A}_{K}^{*} / K^{*} \mathbb{A}_{F}^{*} \longrightarrow \mathbb{C}^{*}$ a (continuous) character of finite order. Fix a number field $L \subset \overline{\mathbb{Q}}$ such that $i_{\infty}(L)$ contains all Hecke eigenvalues $\lambda_{g}(v)$ of $g$ and all values of $\chi$; denote by $\mathfrak{p}$ the prime of $L$ above $p$ induced by $i_{p}$.

Let $V(g)=V_{\mathfrak{p}}(g)$ be the two-dimensional representation of $G_{F}=\operatorname{Gal}(\overline{\mathbb{Q}} / F)$ with coefficients in $L_{\mathfrak{p}}$ attached to $g$ : if $v \nmid \infty p \mathfrak{n}$ is a prime of $F$, then $V(g)$ is unramified at $v$ and

$$
\operatorname{det}\left(1-\operatorname{Fr}(v)_{\text {geom }} X \mid V(g)\right)=1-i_{p}\left(\lambda_{g}(v)\right) X+(N v)^{k-1} X^{2} .
$$

The Tate twist $V=V(g)(k / 2)$ is self-dual in the sense that there exists a skew-symmetric isomorphism $V \stackrel{\sim}{\longrightarrow} V^{*}(1)=\operatorname{Hom}_{L_{\mathfrak{p}}}\left(V, L_{\mathfrak{p}}\right)(1)$.

Denote by $\eta=\eta_{K / F}: \mathbb{A}_{F}^{*} / F^{*} N_{K / F} \mathbb{A}_{K}^{*} \stackrel{\sim}{\longrightarrow}\{ \pm 1\}$ the quadratic character corresponding to the extension $K / F$. 
0.2. - Normalising the reciprocity $\operatorname{map} \operatorname{rec}_{K}: \mathbb{A}_{K}^{*} / K^{*} \longrightarrow \operatorname{Gal}\left(K^{\mathrm{ab}} / K\right)$ so that the local uniformisers correspond to geometric Frobenius elements, we identify $\chi$ with the coresponding Galois character $\chi: G_{K}=\operatorname{Gal}(\overline{\mathbb{Q}} / K) \longrightarrow O_{L}^{*} \subset L_{\mathfrak{p}}^{*}$; put $K_{\chi}=\overline{\mathbb{Q}}^{\operatorname{Ker}(\chi)}$. There are canonical isomorphisms

$$
H_{f}^{1}\left(K, V \otimes \chi^{ \pm 1}\right) \stackrel{\sim}{\longrightarrow}\left(H_{f}^{1}\left(K_{\chi}, V\right) \otimes \chi^{ \pm 1}\right)^{\operatorname{Gal}\left(K_{\chi} / K\right)}=H_{f}^{1}\left(K_{\chi}, V\right)^{\left(\chi^{\mp 1}\right)},
$$

where $H_{f}^{1}(-)$ are the Bloch-Kato Selmer groups and

$$
M^{(\chi)}=\left\{m \in M \mid \forall \sigma \in \operatorname{Gal}\left(K_{\chi} / K\right) \quad \sigma(m)=\chi(\sigma) m\right\},
$$

for any $O_{L}\left[\operatorname{Gal}\left(K_{\chi} / K\right)\right]$-module $M$. The action of the complex conjugation $\rho \in \operatorname{Gal}\left(K_{\chi} / F\right)$ on $H_{f}^{1}\left(K_{\chi}, V\right)$ interchanges the eigenspaces $H_{f}^{1}\left(K_{\chi}, V\right)^{\left(\chi^{ \pm 1}\right)}$, which implies that their dimensions

$$
h_{f}^{1}\left(K, V \otimes \chi^{ \pm 1}\right):=\operatorname{dim}_{L_{\mathfrak{p}}} H_{f}^{1}\left(K, V \otimes \chi^{ \pm 1}\right)
$$

are equal to each other: $h_{f}^{1}(K, V \otimes \chi)=h_{f}^{1}\left(K, V \otimes \chi^{-1}\right)$.

0.3. - Denote by $\pi=\pi(g)$ the (irreducible, cuspidal) automorphic representation of $G L_{2}\left(\mathbb{A}_{F}\right)$ generated by $g$, and by $\theta_{\chi}$ the automorphic representation of $G L_{2}\left(\mathbb{A}_{F}\right)$ generated by the theta series of $\chi$. They are both self-dual, as the central character of $\pi$ (resp., of $\theta_{\chi}$ ) is trivial (resp., is equal to $\eta=\eta_{K / F}$, and $\theta_{\chi} \otimes \eta=\theta_{\chi}$ ).

The Rankin-Selberg $L$-function $L\left(\pi \times \theta_{\chi}, s\right)$ has Euler factors

$$
L_{v}\left(\pi \times \theta_{\chi}, s\right)=\prod_{w \mid v} \operatorname{det}\left(1-\operatorname{Fr}(w)_{\text {geom }}(N w)^{1 / 2-s} \mid(V \otimes \chi)^{I_{w}}\right)^{-1},
$$

where $v \nmid \infty p$ is a prime of $F$ and $w$ a prime of $K$ (cf. [13, 12.6.2.2]). We shall abuse the notation and write $L_{v}(\pi \times \chi, s)$ instead of $L_{v}\left(\pi \times \theta_{\chi}, s\right)$. The complete $L$-function $L(\pi \times \chi, s)=\prod_{v} L_{v}(\pi \times \chi, s)$ is equal to $L\left(\pi \times \chi^{-1}, s\right)$, has holomorphic continuation to $\mathbb{C}$ and a functional equation of the form

$$
\begin{aligned}
& L(\pi \times \chi, s)=\varepsilon(\pi \times \chi, s) L(\pi \times \chi, 1-s), \\
& \varepsilon(\pi \times \chi, s)=c(\pi \times \chi)^{1 / 2-s} \varepsilon\left(\pi \times \chi, \frac{1}{2}\right), \quad \varepsilon\left(\pi \times \chi, \frac{1}{2}\right) \in\{ \pm 1\} .
\end{aligned}
$$

Put

$$
r_{\text {an }}(K, g, \chi):=\operatorname{ord}_{s=1 / 2} L(\pi \times \chi, s)=\operatorname{ord}_{s=1 / 2} \prod_{v \nmid \infty} L_{v}(\pi \times \chi, s)
$$

(the $\Gamma$-factors $L_{v}(\pi \times \chi, s)$ for $v \mid \infty$ take finite non-zero values at $s=1 / 2$ ).

The conjectures of Bloch and Kato ([2], [8]) predict that

$$
r_{\mathrm{an}}(K, g, \chi) \stackrel{?}{=} h_{f}^{1}(K, V \otimes \chi) .
$$

The main result of the present article is the following theorem. 
0.4. Theorem. - Assume that $g \in S_{k}(\mathfrak{n}, 1)$ is potentially p-ordinary, i.e., that there exists a finite solvable extension of totally real number fields $F^{\prime} / F$ such that the base change $B C_{F^{\prime} / F}(\pi(g))$ is equal to $\pi\left(g^{\prime}\right)$, where $g^{\prime}$ is a p-ordinary (cuspidal) Hilbert eigenform over $F^{\prime}$ (equivalently, that there exists a character of finite order $\varphi: \mathbb{A}_{F}^{*} / F^{*} \longrightarrow \overline{\mathbb{Q}}^{*}$ such that the newform associated to $g \otimes \varphi$ is p-ordinary; see [13, 12.5.10]). If $g$ has complex multiplication by a totally imaginary quadratic extension $K^{\prime}$ of $F$, assume, in addition, that $p \neq 2$ and that $K^{\prime} \not \subset K_{\chi}$. Then:

1. If $2 \nmid r_{\mathrm{an}}(K, g, \chi)$, then $2 \nmid h_{f}^{1}(K, V \otimes \chi)$.

2. If $2 \mid r_{\mathrm{an}}(K, g, \chi)$ and if there exists a prime $v \mid p$ of $F$ which does not split in $K / F$ and for which $\pi(g)_{v}=\mathrm{St} \otimes \mu, \mu: F_{v}^{*} \longrightarrow\{ \pm 1\}, \chi_{w}=\mu \circ N_{K_{w} / F_{v}}$, where $w$ is the unique prime of $K$ above $v$ (as $g$ is potentially ordinary, this can occur only if $k=2$; see [13, 12.5.4]), then $2 \mid h_{f}^{1}(K, V \otimes \chi)$.

(The hypothesis in (2) can be interpreted as saying that the Euler factor at $v$ of the p-adic counterpart of $L(\pi \times \chi, s)$ has a trivial zero of odd order at the central point; cf. [13, 12.6.3.10] and $[13,12.6 .4 .3]$.)

0.5. Corollary. - Let $K[\infty] \subset K^{\mathrm{ab}}$ be the union of all ring class fields of $K$ in the sense of $[1,1.1]$ (the Galois group $\operatorname{Gal}(K[\infty] / K)$ is the quotient of $\operatorname{Gal}\left(K^{\mathrm{ab}} / K\right)$ by $\left.\operatorname{rec}_{K}\left(\mathbb{A}_{F}^{*}\right)\right)$. Let $K_{0} / K$ be a finite subextension of $K[\infty] / K$. Assume that $g \in S_{k}(\mathfrak{n}, 1)$ is potentially $p$-ordinary; if $g$ has complex multiplication by a totally imaginary quadratic extension $K^{\prime}$ of $F$, assume, in addition, that $p \neq 2$ and that $K^{\prime} \not \subset K_{0}$. Then

$$
h_{f}^{1}\left(K_{0}, V\right):=\operatorname{dim}_{L_{\mathfrak{p}}} H_{f}^{1}\left(K_{0}, V\right) \geq\left|X^{-}\left(g, K_{0}\right)\right|,
$$

where

$$
X^{ \pm}\left(g, K_{0}\right)=\left\{\chi: \operatorname{Gal}\left(K_{0} / K\right) \longrightarrow \mathbb{C}^{*} \mid \varepsilon\left(\pi(g) \times \chi, \frac{1}{2}\right)= \pm 1\right\}
$$

0.6. Example. - Let $E$ be an elliptic curve over $F$. It is expected that $E$ is modular in the sense that there exists $g \in S_{2}(\mathfrak{n}, 1)$ such that $L_{v}(E / F, s)=L_{v}(\pi(g), s+1 / 2)$, for all primes $v$ of $F$. If this is the case, assume that $E$ has potentially ordinary reduction (= potentially good ordinary or potentially multiplicative reduction) at all primes of $F$ above $p$. If $E$ has complex multiplication by $\mathbb{Q}(\sqrt{-D})$, assume, in addition, that $p \neq 2$ and $F(\sqrt{-D}) \not \subset K_{\chi}$. Theorem 0.4 then implies the following:

1. If $2 \nmid \operatorname{ord}_{s=1} L(E / K, \chi, s)$, then

$$
2 \nmid\left(\operatorname{dim}_{L}\left(E\left(K_{\chi}\right) \otimes L\right)^{\left(\chi^{-1}\right)}+\operatorname{cork}_{O_{L, \mathfrak{p}}}\left(\amalg\left(E / K_{\chi}\right) \otimes O_{L, \mathfrak{p}}\right)^{\left(\chi^{-1}\right)}\right) .
$$

2. If $2 \mid \operatorname{ord}_{s=1} L(E / K, \chi, s)$ and if there exist a prime $v \mid p$ of $F$ which does not split in $K / F$ and a character $\mu: F_{v}^{*} \longrightarrow\{ \pm 1\}$ such that $\chi_{w}=\mu \circ N_{K_{w} / F_{v}}$ and the quadratic twist $E \otimes \mu$ has split multiplicative reduction at $v$, then

$$
2 \mid\left(\operatorname{dim}_{L}\left(E\left(K_{\chi}\right) \otimes L\right)^{\left(\chi^{-1}\right)}+\operatorname{cork}_{O_{L, \mathfrak{p}}}\left(\amalg\left(E / K_{\chi}\right) \otimes O_{L, \mathfrak{p}}\right)^{\left(\chi^{-1}\right)}\right) .
$$

There is an obvious variant of this statement when $E$ is replaced by an abelian variety $A_{0}$ with $O_{L_{0}} \hookrightarrow \operatorname{End}_{F}\left(A_{0}\right)$, where $L_{0}$ is a totally real number field of degree $\left[L_{0}: \mathbb{Q}\right]=\operatorname{dim}\left(A_{0}\right)$. 
0.7. Example [7]. - Let $q \neq 2$ be a prime number and $F_{0}$ a totally real number field such that $F_{0} \cap \mathbb{Q}\left(\mu_{q \infty}\right)=\mathbb{Q}$. Let $a \in O_{F_{0}}-\{0\}$ be an element satisfying $a \notin F_{0}^{* q}$ and, for each finite prime $v_{0}$ of $F_{0}$ not dividing $q$, $\operatorname{ord}_{v_{0}}(a)<q$. For each $r \geq 1$, put $F_{r}=F_{0} \mathbb{Q}\left(\mu_{q^{r}}\right)^{+}$, $K_{r}=F_{0}\left(\mu_{q^{r}}\right)$; then $\operatorname{Gal}\left(K_{r} / F_{0}\right) \stackrel{\sim}{\longrightarrow}\left(\mathbb{Z} / q^{r} \mathbb{Z}\right)^{*}$ and $\operatorname{Gal}\left(K_{r}\left(\sqrt[q^{r}]{a}\right) / K_{r}\right) \stackrel{\sim}{\longrightarrow} \mathbb{Z} / q^{r} \mathbb{Z}$. Fix an injective character $\chi_{r}: \operatorname{Gal}\left(K_{r}(\sqrt[a]{a}) / K_{r}\right) \longrightarrow \overline{\mathbb{Q}}^{*}$; then $\chi_{r}$ (more precisely, $\chi_{r} \circ \operatorname{rec}_{K_{r}}$ ) factors through $\mathbb{A}_{K_{r}}^{*} / K_{r}^{*} \mathbb{A}_{F_{r}}^{*}$.

Let $g_{0} \in S_{k}\left(\mathfrak{n}_{0}, 1\right)$ be a cuspidal Hilbert newform over $F_{0}$ of parallel weight $k$, trivial character and level $\mathfrak{n}_{0}$; put $V=V_{\mathfrak{p}}\left(g_{0}\right)(k / 2)$. For $r \geq 1$, denote by $g_{r}$ the corresponding base change newform over $F_{r}$ (i.e., $B C_{F_{r} / F}\left(\pi\left(g_{0}\right)\right)=\pi\left(g_{r}\right)$ ). An easy exercise in group theory (see $\$ 3.1$ below) shows that, for each $r \geq 1$,

$$
H_{f}^{1}\left(F_{0}\left(\sqrt[q^{r}]{a}\right), V\right)=H_{f}^{1}\left(F_{0}, V\right) \oplus \bigoplus_{s=1}^{r} H_{f}^{1}\left(K_{s}, V \otimes \chi_{s}\right)
$$

0.8. Theorem. - In the notation of 0.7, assume that $\left(\forall v_{0} \mid q\right) \pi\left(g_{0}\right)_{v_{0}}$ is a principal series representation and that $\left(\forall v_{0} \mid a\right) \pi\left(g_{0}\right)_{v_{0}}$ is not supercuspidal. Put $\mathfrak{n}_{0}^{(a q)}=\mathfrak{n}_{0} /\left(\mathfrak{n}_{0},(a q)^{\infty}\right)$ and $d=(-1)^{\left[F_{0}: \mathbb{Q}\right]} N\left(\mathfrak{n}_{0}^{(a q)}\right) \in \mathbb{Z}-\{0\}$. Then:

1. For each $r \geq 1, \varepsilon\left(\pi\left(g_{r}\right) \times \chi_{r}, \frac{1}{2}\right)=\left(\frac{d}{q}\right)$. (See [7, Thm. 6] for a special case.)

2. Assume that $g_{0}$ is potentially $p$-ordinary. If $g_{0}$ has complex multiplication by a totally imaginary quadratic extension $K_{0}^{\prime}$ of $F_{0}$, assume that $p \neq 2$ and that $(q \equiv 1(\bmod 4)$ or $K_{0}^{\prime} \neq F_{0}(\sqrt{-q})$ ) (the latter condition is automatically satisfied if $2 \nmid\left[F_{0}: \mathbb{Q}\right]$ ). If $\left(\frac{d}{q}\right)=-1$, then

$$
\begin{aligned}
\forall r \geq 0 \quad & h_{f}^{1}\left(F_{0}\left(\sqrt[q^{r}]{a}\right), V\right)-h_{f}^{1}\left(F_{0}, V\right) \geq r \\
& h_{f}^{1}\left(F_{0}\left(\sqrt[q^{r}]{a}\right), V\right)-h_{f}^{1}\left(F_{0}, V\right) \equiv r(\bmod 2), \\
& h_{f}^{1}\left(F_{0}\left(\mu_{q^{r}}, \sqrt[q^{r}]{a}\right), V\right)-h_{f}^{1}\left(F_{0}, V\right) \geq q^{r}-1 .
\end{aligned}
$$

0.9. Corollaire. - In the notation of 0.7 , assume that $E$ is a modular elliptic curve over $F_{0}$ such that for each prime $v_{0}$ of $F_{0}$ above $q$ (resp., dividing a) there exists a quadratic twist of $E$ with good reduction (resp., semistable reduction) at $v_{0}$. If $E$ has complex multiplication, assume that $p \neq 2$ and that $\left(q \equiv 1(\bmod 4)\right.$ or $\left.F_{0} \otimes \operatorname{End}_{\overline{\mathbb{Q}}}(E) \neq F_{0}(\sqrt{-q})\right)$ (the latter condition is automatically satisfied if $\left.2 \nmid\left[F_{0}: \mathbb{Q}\right]\right)$. Assume, finally, that $\left(\frac{d}{q}\right)=-1$, where $d=(-1)^{\left[F_{0}: \mathbb{Q}\right]} N\left(\operatorname{cond}(E)^{(a q)}\right)$. Then, for each prime number $p$ such that $E$ has potentially ordinary reduction at all primes of $F_{0}$ above $p$, the ranks

$$
s_{p}(E /-):=\operatorname{rk}_{\mathbb{Z}} E(-)+\operatorname{cork}_{\mathbb{Z}_{p}} \amalg(E /-)\left[p^{\infty}\right]
$$

satisfy

$$
\begin{aligned}
\forall r \geq 0 \quad & s_{p}\left(E / F_{0}\left(\sqrt[q^{r}]{a}\right)\right)-s_{p}\left(E / F_{0}\right) \geq r, \\
& s_{p}\left(E / F_{0}\left(\sqrt[q^{r}]{a}\right)\right)-s_{p}\left(E / F_{0}\right) \equiv r(\bmod 2), \\
& s_{p}\left(E / F_{0}\left(\mu_{q^{r}}, \sqrt[q^{r}]{a}\right)\right)-s_{p}\left(E / F_{0}\right) \geq q^{r}-1 .
\end{aligned}
$$


0.10. Remarks. - (1) The proof of Theorem 0.4 does not use any explicit formula for the global root number $\varepsilon\left(\pi(g) \times \chi, \frac{1}{2}\right)$ (in fact, such a formula is not available in general). Instead, the local root numbers enter the picture indirectly, through their representation-theoretical characterisation ([18], [19, Thm. 2], [17]) that appears in a refinement of the method of Cornut and Vatsal ([1]).

(2) A suitable strengthening of the methods of [12] or [5] would allow us to drop the assumption of potentially ordinary reduction at $p$ in Example 0.6 (if the order of $\chi$ is a power of $p$, this can be done, in many cases, by combining [12, Thm. 6.4], [5, Thm. 4.3] and [16, Thm. 1]).

(3) In the special case $p=q$ there are more elementary approaches to Corollary 0.9 ([12, Thm. 7.1], [6, Prop. 4.13], [3, Thm. 4.2, 4.6]), which do not require $E$ to be modular but instead assume that $s_{p}\left(E / F_{0}\left(\mu_{p}\right)\right) \equiv \operatorname{ord}_{s=1} L\left(E / F_{0}\left(\mu_{p}\right), s\right) \quad(\bmod 2)$, which under fairly general circumstances follows from [16, Thm. 1].

(4) If $\chi^{2}=1$, then $\chi=\varphi \circ N_{K / F}$, where $\varphi: \mathbb{A}_{F}^{*} / F^{*} \longrightarrow\{ \pm 1\}$, and

$$
H_{f}^{1}(K, V \otimes \chi)=H_{f}^{1}(F, V \otimes \varphi) \oplus H_{f}^{1}\left(F, V \otimes \varphi \eta_{K / F}\right) .
$$

In many cases [13, Thm. 12.2.3] applies to $g \otimes \varphi$ and $g \otimes \varphi \eta_{K / F}$, yielding

$$
r_{\text {an }}(K, g, \chi) \equiv h_{f}^{1}(K, V \otimes \chi)(\bmod 2) .
$$

\section{Proof of Theorem 0.4}

In this section we prove Theorem 0.4 , following closely the arguments in [13, §12.9]. The main difference is that the non-vanishing results of [4, §4] are replaced by their strengthening proved in [1]. We are going to use the notation from [1] and [13, ch. 12].

Assume that $g \in S_{k}(\mathfrak{n}, 1)$ is potentially $p$-ordinary and, if $g$ has complex multiplication by a totally imaginary quadratic extension $K^{\prime}$ of $F$, that $p \neq 2$ and $K^{\prime} \not \subset K_{\chi}$. Put $\pi=\pi(g)$, $\eta=\eta_{K / F}, \varepsilon_{v}=\varepsilon\left(\pi_{v} \times \chi_{v}, \frac{1}{2}\right) \in\{ \pm 1\}$ and $\varepsilon=\varepsilon\left(\pi \times \chi, \frac{1}{2}\right)=\prod_{v} \varepsilon_{v}$. Note that $\varepsilon_{v}$ does not depend on the choice of an additive character, by the self-duality of $V \otimes \operatorname{Ind}_{K / F}(\chi)$ $([14,2.2 .1(1)])$.

\subsection{Reduction steps}

1.1.1. - Let $\Sigma_{p}$ (resp., $\left.\Sigma_{\infty}=\left\{\tau_{1}, \ldots, \tau_{d}\right\}, d=[F: \mathbb{Q}]\right)$ be the set of all primes of $F$ above $p$ (resp., above $\infty$ ). Put

$$
\begin{aligned}
& \Sigma_{\chi}=\Sigma_{\chi}(g)=\left\{v \in \Sigma_{p} \mid v \text { does not split in } K / F,\right. \\
& \left.\qquad \pi_{v}=\operatorname{St} \otimes \mu, \mu: F_{v}^{*} \longrightarrow\{ \pm 1\}, \chi_{w}=\mu \circ N_{K_{w} / F_{v}}\right\}
\end{aligned}
$$

(where $w$ is the unique prime of $K$ above $v$ ). As $g$ is potentially $p$-ordinary, $\pi_{v}$ is not supercuspidal for any $v \in \Sigma_{p}$, which implies that

$$
\forall v \in \Sigma_{p} \quad \eta_{v}(-1) \varepsilon_{v}= \begin{cases}-1, & v \in \Sigma_{\chi} \\ 1, & v \notin \Sigma_{\chi}\end{cases}
$$

(see $[13,12.6 .2 .4])$. At the archimedean primes,

$$
\forall v \in \Sigma_{\infty} \quad \eta_{v}(-1)=-1, \quad \varepsilon_{v}=1 .
$$


1.1.2. Reduction to the case $k=2$. - Assume that $k>2$ and that Theorem 0.4 has been proved for $k=2$. As in $[13,12.9 .5 .1]$, let $g^{\prime} \in S_{2}\left(\mathfrak{n}^{\prime}, 1\right)$ be a weight 2 specialisation of the twisted Hida family containing a twist of the $p$-stabilisation of a $p$-ordinary twist of $g$; put $\pi^{\prime}=\pi\left(g^{\prime}\right), V^{\prime}=V_{\mathfrak{p}}\left(g^{\prime}\right)(1)$. As $k>2$, we have $\Sigma_{\chi}(g)=\varnothing([13$, Lemma 12.5.4]). According to [13, 12.7.14.5(v), (ii), (iii)] (combined with (2.2.3.1) below),

(1.1.2.1) $g$ has complex multiplication by $K^{\prime} \Longleftrightarrow g^{\prime}$ has complex multiplication by $K^{\prime}$;

$$
\begin{gathered}
r_{\mathrm{an}}(K, g, \chi)=r_{\mathrm{an}}(K, g, \chi)+\left|\Sigma_{\chi}(g)\right| \equiv r_{\mathrm{an}}\left(K, g^{\prime}, \chi\right)+\left|\Sigma_{\chi}\left(g^{\prime}\right)\right|(\bmod 2) \\
h_{f}^{1}(K, V \otimes \chi)=h_{f}^{1}(K, V \otimes \chi)+\left|\Sigma_{\chi}(g)\right| \equiv h_{f}^{1}\left(K, V^{\prime} \otimes \chi\right)+\left|\Sigma_{\chi}\left(g^{\prime}\right)\right|(\bmod 2) .
\end{gathered}
$$

As Theorem 0.4 holds for $g^{\prime}$ by assumption, the statements 1.1.2.1-3 imply that it also holds for $g$ (in fact, one can always choose $g^{\prime}$ satisfying $\Sigma_{\chi}\left(g^{\prime}\right)=\varnothing$, by $[13,12.7 .10]$ ).

1.1.3. Further reduction in the case $k=2$ (cf. $[13,12.10 .1])$. - From now on, until the end of $\S 1, k=2$. Assume that Theorem 0.4 has been proved for $k=2$ under the following additional assumption:

$$
\exists \Sigma \subseteq \Sigma_{p}, \Sigma \neq \varnothing \quad(-1)^{\left|\Sigma \cap \Sigma_{\chi}\right|-1}=\varepsilon\left(\pi \times \chi, \frac{1}{2}\right)=\varepsilon .
$$

There is precisely one case in which the assumptions of Theorem $0.4\left(\varepsilon=-1\right.$ or $\left.\left|\Sigma_{\chi}\right| \neq 0\right)$ are satisfied, but 1.1.3.1 is not:

$$
\left|\Sigma_{\chi}\right|=\left|\Sigma_{p}\right|=1, \quad \varepsilon=-1 .
$$

In this case fix a totally real cyclic extension $F_{1} / F$ of odd order $n>1$ in which the unique prime $v \in \Sigma_{p}$ splits completely and put $K_{1}=F_{1} K, \chi_{1}=\chi \circ N_{K_{1} / K}: \mathbb{A}_{K_{1}}^{*} / K_{1}^{*} \mathbb{A}_{F_{1}}^{*} \longrightarrow \mathbb{C}^{*}$ (thus $\left.\left(K_{1}\right)_{\chi_{1}}=F_{1} K_{\chi}\right), \pi_{1}=B C_{F_{1} / F}(\pi)=\pi\left(g_{1}\right), \Delta=\operatorname{Gal}\left(F_{1} / F\right)=\operatorname{Gal}\left(K_{1} / K\right)$, $\widehat{\Delta}=\operatorname{Hom}\left(\Delta, \mathbb{C}^{*}\right)$. As $g$ is potentially $p$-ordinary, so is the base change form $g_{1}$ over $F_{1}$. Furthermore, if $g$ has complex multiplication by $K^{\prime}\left(\left[K^{\prime}: F\right]=2\right)$, then $g_{1}$ has complex multiplication by $F_{1} K^{\prime}$, and

$$
K^{\prime} \not \subset K_{\chi} \Longleftrightarrow F_{1} K^{\prime} \not \subset F_{1} K_{\chi}=\left(K_{1}\right)_{\chi_{1}} .
$$

We have

$$
\varepsilon\left(\pi_{1} \times \chi_{1}, \frac{1}{2}\right)=\prod_{\alpha \in \widehat{\Delta}} \varepsilon\left(\pi \times\left(\theta_{\chi} \otimes \alpha\right), \frac{1}{2}\right), \quad h_{f}^{1}\left(K_{1}, V \otimes \chi_{1}\right)=\sum_{\alpha \in \widehat{\Delta}} h_{f}^{1}(K, V \otimes \chi \alpha) .
$$

As

$$
\varepsilon\left(\pi \times\left(\theta_{\chi} \otimes \alpha^{-1}\right), \frac{1}{2}\right)=\varepsilon\left(\pi \times\left(\theta_{\chi} \otimes \alpha\right), \frac{1}{2}\right)^{-1}
$$

by the functional equation, it follows that

$$
r_{\text {an }}\left(K, g_{1}, \chi_{1}\right) \equiv r_{\text {an }}(K, g, \chi)(\bmod 2) .
$$

Similarly,

$$
\begin{aligned}
& \begin{aligned}
h_{f}^{1}(K, V \otimes \chi \alpha) & =h_{f}^{1}\left(K, V^{*}(1) \otimes \chi^{-1} \alpha^{-1}\right)=h_{f}^{1}\left(K, V \otimes \chi^{-1} \alpha^{-1}\right) \quad([13,12.5 .9 .5(\mathrm{iv})]) \\
& =h_{f}^{1}\left(K, V \otimes \chi \alpha^{-1}\right)
\end{aligned} \\
& 4^{\text {e }} \text { SÉRIE - TOME } 41-2008-\mathrm{N}^{\mathrm{o}} 6
\end{aligned}
$$


(the last equality follows from the fact that the action of the complex conjugation $\rho \in$ $\operatorname{Gal}\left(F_{1} K_{\chi} / F\right)$ interchanges the $\chi \alpha$ - and $\chi^{-1} \alpha$-eigenspaces for the action of $\operatorname{Gal}\left(F_{1} K_{\chi} / K\right)$ on $\left.H_{f}^{1}\left(F_{1} K_{\chi}, V\right)\right)$, which implies that

$$
h_{f}^{1}\left(K_{1}, V \otimes \chi_{1}\right) \equiv h_{f}^{1}(K, V \otimes \chi)(\bmod 2) .
$$

Finally,

$$
\Sigma_{\chi_{1}}\left(g_{1}\right)=\left\{v_{1} \mid v, v \in \Sigma_{\chi}(g)\right\} .
$$

Putting (1.1.3.2-1.1.3.5) together, we obtain that Theorem 0.4 holds for $g$ iff it holds for $g_{1}$. However, $g_{1}$ satisfies (1.1.3.1), as there are $n>1$ primes above $p$ in $F_{1}$. This concludes the proof of the reduction step to the case when (1.1.3.1) holds.

\subsection{Passage to a Shimura curve}

1.2.1. - Thanks to 1.1, we can (and will) assume that $k=2$ and that there exists a nonempty subset $\Sigma=\left\{P_{1}, \ldots, P_{s}\right\} \subseteq \Sigma_{p}$ for which (1.1.3.1) holds; fix such $\Sigma$ (in fact, we can always take $s=1$ or $s=2)$. Our goal is to show that $r_{\text {an }}(K, g, \chi) \equiv h_{f}^{1}(K, V \otimes \chi)(\bmod 2)$.

\subsubsection{Choice of a quaternion algebra. - As}

$$
\begin{aligned}
\varepsilon & =\prod_{v} \varepsilon_{v}=\prod_{v} \eta_{v}(-1) \varepsilon_{v} \\
& =\prod_{v \in \Sigma_{\infty}} \prod_{v \in \Sigma} \prod_{v \notin \Sigma \cup \Sigma_{\infty}} \eta_{v}(-1) \varepsilon_{v}=(-1)^{[F: \mathbb{Q}]}(-1)^{\left|\Sigma \cap \Sigma_{\chi}\right|} \prod_{v \notin \Sigma \cup \Sigma_{\infty}} \eta_{v}(-1) \varepsilon_{v}
\end{aligned}
$$

(by (1.1.1.1-1.1.1.2)), the condition (1.1.3.1) implies that

$$
\prod_{v \notin \Sigma \cup \Sigma_{\infty}} \eta_{v}(-1) \varepsilon_{v}=(-1)^{[F: \mathbb{Q}]-1} .
$$

This means that there exists a (unique) quaternion algebra $B$ over $F$ with local invariants

$$
\operatorname{inv}_{v}(B)= \begin{cases}1, & v \in \Sigma \cup\left\{\tau_{1}\right\} \\ -1, & v \in \Sigma \Sigma_{\infty}-\left\{\tau_{1}\right\} \\ \eta_{v}(-1) \varepsilon_{v}, & v \notin \Sigma \cup \Sigma_{\infty} .\end{cases}
$$

1.2.3. Proposition. - (1) There exists a (unique) irreducible (cuspidal) automorphic representation $\pi^{\prime}$ of $B_{\mathbb{A}}^{*}$ such that $\pi=J L\left(\pi^{\prime}\right)$ is associated to $\pi^{\prime}$ by the Jacquet-Langlands correspondence. The representation $\pi^{\prime}$ has trivial central character, $\pi_{v}^{\prime}=1$ if $v \in \Sigma_{\infty}-\left\{\tau_{1}\right\}$, and $\pi_{\tau_{1}}^{\prime}$ is the weight 2 holomorphic discrete series.

(2) There exists an F-embedding $t: K \hookrightarrow B$; fix such $t$.

(3) $\forall v \notin \Sigma \cup \Sigma_{\infty}$ there exists a non-zero $t_{v}\left(K_{v}^{*}\right)$-invariant linear form $\ell_{v}: \pi_{v}^{\prime} \longrightarrow \mathbb{C}\left(\chi_{v}^{-1}\right)$.

Proof. - (1) At each finite prime $v$ of $F$ at which $\pi_{v}$ is a principal series representation we have $\eta_{v}(-1) \varepsilon_{v}=1$ ([13, 12.6.2.4(i)]), hence $B$ is unramified at $v$, by construction. This implies that $\pi$ lies in the image of the Jacquet-Langlands correspondence. The central characters of $\pi$ and $\pi^{\prime}$ coincide and the archimedean behaviour of $\pi^{\prime}$ follows from the corresponding local correspondence. 
(2) At each finite prime $v$ of $F$ which splits in $K / F$ we have $\eta_{v}(-1) \varepsilon_{v}=1$ ([13, 12.6.2.4]); thus $B$ is ramified only at primes of $F$ that do not split in $K / F$, which implies the existence of $t$.

(3) By construction, $\operatorname{inv}_{v}(B)=\eta_{v}(-1) \varepsilon_{v}$ for each $v \notin \Sigma \cup \Sigma_{\infty}$; we apply the fundamental results of [18] and [19, Thm. 2] (see also [17]) (note that, if $\operatorname{inv}_{v}(B)=1$, then $\operatorname{dim}\left(\pi_{v}^{\prime}\right)=$ $\left.\operatorname{dim}\left(\pi_{v}\right)=\infty\right)$.

1.2.4. Proposition. Choice of an order. - There exists an $O_{F}$-order $R \subset B$ such that $\forall v \notin$ $\Sigma \cup \Sigma_{\infty}, \ell_{v}\left(\left(\pi_{v}^{\prime}\right)^{R_{v}^{*}}\right) \neq 0 ; \forall v \in \Sigma, R_{v}$ is an Eichler order of level $o\left(\pi_{v}^{\prime}\right)=o\left(\pi_{v}\right)$ (hence $\left.\operatorname{dim}\left(\pi_{v}^{\prime}\right)^{R_{v}^{*}}=1\right)$.

Proof. - Fix any $O_{F}$-order $R_{0} \subset B$. There exists a finite set $S_{0} \supset \Sigma_{\infty}$ of primes of $F$ such that, for each $v \notin S_{0}$, the following conditions hold: $R_{0, v}$ is a maximal $O_{F, v}$-order in $B_{v} \stackrel{\sim}{\longrightarrow} M_{2}\left(F_{v}\right), t_{v}^{-1}\left(R_{0, v}\right)=O_{K, v}, \chi_{v}$ is unramified and $o\left(\pi_{v}^{\prime}\right)=o\left(\pi_{v}\right)=0\left(\Longleftrightarrow \pi_{v}\right.$ is an unramified principal series representation). This implies, by [9, 2.3], that $\ell_{v}\left(\left(\pi_{v}^{\prime}\right)^{R_{v}^{*}}\right) \neq 0$ for all $v \notin S_{0}$. On the other hand, for each $v \in S_{0}-\left(\Sigma \cup \Sigma_{\infty}\right)$, there exists a vector $x_{v} \in \pi_{v}^{\prime}$ such that $\ell_{v}\left(x_{v}\right) \neq 0$. As the central character of $\pi_{v}^{\prime}$ is trivial, there exists an $O_{F, v}$-order $R(v) \subset B_{v}$ such that $x_{v} \in\left(\pi_{v}^{\prime}\right)^{R(v)^{*}}$. For $v \in \Sigma$, let $R(v) \subset B_{v}$ be any Eichler order of level $o\left(\pi_{v}\right)$. The $O_{F}$-order $R \subset B$ given by its localisations

$$
R_{v}= \begin{cases}R_{0, v}, & v \notin S_{0} \\ R(v), & v \in S_{0}-\Sigma_{\infty}\end{cases}
$$

has the required properties.

1.2.5. Shimura curve. - Fix an $O_{F}$-order $R \subset B$ satisfying Proposition 1.2 .4 and put $H=$ $\widehat{R}^{*}$. From now on, we shall work with the Shimura curve $N_{H}^{*}$ (in the notation of $[15,1.4]$ and $[1,2.1 .2])$. By construction,

$$
\pi^{\prime H}=\pi_{\infty}^{\prime} \otimes \bigotimes_{v \nmid \infty}^{\prime}\left(\pi_{v}^{\prime}\right)^{R_{v}^{*}} \neq 0
$$

\subsection{CM points}

1.3.1. - As in [1, 2.2], fix an isomorphism $B_{\tau_{1}} \stackrel{\sim}{\longrightarrow} M_{2}(\mathbb{R})$ and denote by $z \in \mathbb{C}, \operatorname{Im}(z)>0$ the unique point fixed by $t\left(K^{*}\right) \subset B^{*} \subset B_{\tau_{1}}^{*} \stackrel{\sim}{\longrightarrow} G L_{2}(\mathbb{R})$. Let

$$
\mathcal{C}=\left\{\left[z, b_{1} \cdots b_{s}\right]_{H} \mid b_{i} \in B_{P_{i}}^{*}\right\} \subset C M\left(N_{H}, K\right)
$$

be the $\left(P_{1} \cdots P_{s}\right)$-isogeny class of the CM point $[z, 1]_{H}$ on the curve $N_{H}^{*}$.

Recall that the conductor of a CM point $x=[z, b]_{H} \in C M\left(N_{H}, K\right)$ is the non-zero ideal $c(x) \subset O_{F}$ satisfying

$$
\forall v \notin \Sigma_{\infty} \quad t_{v}^{-1}\left(b_{v} R_{v} b_{v}^{-1}\right)=O_{F, v}+c(x) O_{K, v} .
$$

Similarly, the conductor of $\chi$ is the smallest non-zero ideal $c(\chi) \subset O_{F}$ (w.r.t. divisibility) such that

$$
\forall v \notin \Sigma_{\infty} \quad \chi_{v}\left(\left(O_{F, v}+c(\chi) O_{K, v}\right)^{*}\right)=1 .
$$


1.3.2. Lemma. $-\forall v \notin \Sigma \cup \Sigma_{\infty}, \forall x \in \mathcal{C}, \operatorname{ord}_{v}(c(\chi)) \leq \operatorname{ord}_{v}(c(x))$ (the R.H.S. depends only on $\mathcal{C}$, by $[1,(2.2 .4 .1)])$.

Proof. - There exists $x_{v} \in\left(\pi_{v}^{\prime}\right)^{R_{v}^{*}}$ such that $\ell_{v}\left(x_{v}\right) \neq 0$. As

$$
\forall a \in K_{v}^{*} \quad \ell_{v}\left(\pi_{v}^{\prime}\left(t_{v}(a)\right) x_{v}\right)=\chi_{v}^{-1}(a) \ell_{v}\left(x_{v}\right)
$$

it follows that $\chi_{v}\left(t_{v}^{-1}\left(R_{v}^{*}\right)\right)=1$; but $t_{v}^{-1}\left(R_{v}\right)=O_{F, v}+c(x) O_{K, v}$, by definition.

1.3.3. Corollary-Definition. - Put $c_{0}=c(\chi) /\left(c(\chi),\left(P_{1} \cdots P_{s}\right)^{\infty}\right)$ and $c=c(x) /(c(x)$, $\left.\left(P_{1} \cdots P_{s}\right)^{\infty}\right)$ (which is independent of the choice of $x \in \mathcal{C}$ ). Then $c_{0} \mid c$ and $c(\chi)=$ $c_{0} P_{1}^{n_{1}} \cdots P_{s}^{n_{s}}\left(n_{i} \in \mathbb{N}\right)$. As $c_{0} \mid c$, $\chi$ is a character of $G^{(c)}=\operatorname{Gal}\left(K\left[c P^{\infty}\right] / K\right)$, in the notation of $[1,1.2 .2]$; let $\chi_{0}: G_{0}^{(c)} \longrightarrow \mathbb{C}^{*}$ be the restriction of $\chi$ to the torsion subgroup $G_{0}^{(c)}=\left(G^{(c)}\right)_{\text {tors }}$ of $G^{(c)}$.

1.3.4. Lemma. - For each $m \in \mathbb{N}$, there exists a CM point $x_{m} \in \mathcal{C}$ which is good in the sense of $[1,4.2 .3]$ and satisfies $\left(P_{1} \cdots P_{s}\right)^{m} \mid c\left(x_{m}\right)$.

Proof. - Let

$$
X: \prod_{i=1}^{s} \mathcal{P}_{a_{i}}\left(\mathcal{T}_{i}\right) \rightarrow \mathcal{C}
$$

be the $\prod_{i=1}^{s} K_{P_{i}}^{*}$-equivariant surjection from [1, (4.2.2.3)]. For each $i=1, \ldots, s$ choose a path $\gamma_{i}=\left(y_{0} \rightarrow \cdots \rightarrow y_{a_{i}}\right) \in \mathcal{P}_{a_{i}}\left(\mathcal{T}_{i}\right)$ such that $\mathscr{L}_{i}\left(y_{0}\right)=m$ and $\mathscr{L}_{i}\left(y_{a_{i}}\right)=m+a_{i}$; the CM point $x_{m}=X\left(\gamma_{1}, \ldots, \gamma_{s}\right)$ has the required properties.

\subsection{Quotient of the Jacobian of the Shimura curve}

1.4.1. - As in $[15,1.16]$, denote by $J\left(N_{H}^{*}\right)$ the Jacobian of the curve $N_{H}^{*}$. As recalled in [1, 2.1.7] and [15, 1.18], there exists an isogeny $J\left(N_{H}^{*}\right) \longrightarrow A_{0}^{a} \times A_{1}$ (defined over $F$ ), where: $A_{0}$ is an $F$-simple abelian variety with $\operatorname{End}_{F}\left(A_{0}\right)=O_{L_{0}}, L_{0}$ is a totally real number field of degree $\left[L_{0}: \mathbb{Q}\right]=\operatorname{dim}\left(A_{0}\right)$, there is a unique embedding $\sigma_{0}: L_{0} \hookrightarrow \mathbb{R}$ such that the $L$-function $L\left(\sigma_{0} A_{0} / F, s\right)=L\left(\sigma_{0} h^{1}\left(A_{0}\right), s\right)$ (in the notation of [14, 0.0]) is equal (Euler factor by Euler factor) to $L\left(\pi^{\prime}, s-1 / 2\right)=L(\pi, s-1 / 2), a=\operatorname{dim} \bigotimes_{v \nmid \infty}^{\prime}\left(\pi_{v}^{\prime}\right)^{R_{v}^{*}} \geq 1$ and $\operatorname{Hom}_{F}\left(A_{0}, A_{1}\right)=0$ (in the notation of [1, 2.1.7], $\pi^{\prime}=\pi\left(\sigma_{0}\right)$ ). As $\sigma_{0}\left(L_{0}\right)$ is the field generated over $\mathbb{Q}$ by the Hecke eigenvalues of $g$, there is a unique embedding $i: L_{0} \hookrightarrow L$ satisfying $\left.i_{\infty}\right|_{L} \circ i=\sigma_{0}$.

For $j=1, \ldots, a$, denote by $\operatorname{pr}_{j}: A_{0}^{a} \longrightarrow A_{0}$ the projection on the $j$-th factor.

1.4.2. - Put $S=\left\{v \mid c d_{K / F}, v \neq P_{1}, \ldots, P_{s}\right\}$. According to Proposition 1.2.4,

$$
\forall v \in S \quad \ell_{v}\left(\left(\pi_{v}^{\prime}\right)^{R_{v}^{*}}\right) \neq 0
$$

Denote by

$$
\ell_{S}=\bigotimes_{v \in S} \ell_{v}: \bigotimes_{v \in S} \pi_{v}^{\prime} \longrightarrow \mathbb{C}\left(\chi_{S}^{-1}\right)
$$

the tensor product of the linear forms $\ell_{v}(v \in S)$; above, $\chi_{S}=\prod_{v \in S} \chi_{v}: \prod_{v \in S} K_{v}^{*} \longrightarrow \mathbb{C}^{*}$. 
1.4.3. - As in [1, 2.4.3], put $A=O_{L} \otimes_{O_{L_{0}}} A_{0}$. As explained in [1, 2.1.7], $\Gamma\left(A, \Omega_{A / F}^{1}\right)$ is a free $L \otimes_{\mathbb{Q}} F$-module of rank one and the projection $J\left(N_{H}^{*}\right) \longrightarrow A_{0}^{a} \times A_{1} \longrightarrow A_{0}^{a}$ induces an isomorphism

$$
\Gamma\left(A, \Omega_{A / F}^{1}\right)^{a} \otimes_{F, \tau_{1}} \mathbb{C} \stackrel{\sim}{\longrightarrow} \bigoplus_{\sigma: L \hookrightarrow \mathbb{C}} \bigotimes_{v \nmid \infty}^{\prime}\left({ }^{\sigma} \pi_{v}^{\prime}\right)^{R_{v}^{*}} .
$$

In particular, there is a natural $\widehat{B}^{*}$-equivariant projection on the term corresponding to $\sigma=\left.i_{\infty}\right|_{L}$ :

$$
r: \Gamma\left(A, \Omega_{A / F}^{1}\right)^{a} \otimes_{F, \tau_{1}} \mathbb{C} \rightarrow \bigotimes_{v \nmid \infty}^{\prime}\left(\pi_{v}^{\prime}\right)^{R_{v}^{*}} .
$$

The linear form $\ell_{S}$ induces a linear map

$$
\ell_{S} \otimes \mathrm{id}: \bigotimes_{v \nmid \infty}^{\prime} \pi_{v}^{\prime} \longrightarrow\left(\bigotimes_{v \notin \Sigma_{\infty} \cup S}^{\prime} \pi_{v}^{\prime}\right) \otimes \chi_{S}^{-1}
$$

and it follows from (1.4.2.1) that there exists $j \in\{1, \ldots, a\}$ such that

$$
\left(\ell_{S} \otimes \text { id }\right) \circ r \circ \operatorname{pr}_{j}^{*}\left(\Gamma\left(A, \Omega_{A / F}^{1}\right) \otimes_{F, \tau_{1}} \mathbb{C}\right) \neq 0 .
$$

In the notation of [1, 2.1.3], let $\alpha: N_{H}^{*} \longrightarrow A_{0}$ be the morphism (defined over $F$ ) given by

$$
\alpha: N_{H}^{*} \stackrel{\iota_{H}}{\longrightarrow} J\left(N_{H}^{*}\right) \longrightarrow A_{0}^{a} \times A_{1} \longrightarrow A_{0}^{a} \stackrel{\mathrm{pr}_{j}}{\longrightarrow} A_{0} .
$$

\subsection{End of the proof}

1.5.1. - Using Lemma 1.3.4, choose a sequence of good CM points $\left\{x_{m}\right\} \subseteq \mathcal{C}$ satisfying $\left(P_{1} \cdots P_{s}\right)^{m} \mid c\left(x_{m}\right)$ for all $m \in \mathbb{N}$. By (1.4.3.1), the main non-vanishing result from [1, Theorem 4.3.1] applies to the sequence of points $\left\{\alpha\left(x_{m}\right)\right\} \subset A_{0}\left(K\left[c P^{\infty}\right]\right)$ and the character $\chi_{0}$ : if $m \in \mathbb{N}$ is large enough, then there exists a character $\chi_{m}: G^{(c)} \longrightarrow \overline{\mathbb{Q}}^{*}$ of finite order with the following properties:

$$
\begin{aligned}
& \text { the restriction of } \chi_{m} \text { to } G_{0}^{(c)} \text { is equal to } \chi_{0} ; \\
& \left.c\left(\chi_{m}\right) c_{0}^{-1}=c\left(x_{m}\right) c^{-1} \text { (hence }\left(P_{1} \cdots P_{s}\right)^{m} \mid c\left(\chi_{m}\right)\right) ; \\
& e_{\bar{\chi}_{m}}\left(1 \otimes \alpha\left(x_{m}\right)\right) \neq 0 \in \overline{\mathbb{Q}} \otimes_{O_{L_{0}}} A_{0}\left(K\left[c P^{\infty}\right]\right) .
\end{aligned}
$$

The assumptions in Theorem 0.4 imply that $g$ does not have complex multiplication by any totally imaginary quadratic extension of $F$ contained in $K_{\chi_{m}}$, which means that the main result of [15, Thm. 3.2] applies to the non-torsion point $e_{\bar{\chi}_{m}}\left(1 \otimes \alpha\left(x_{m}\right)\right)$, yielding

$$
h_{f}^{1}\left(K, V \otimes \chi_{m}^{ \pm 1}\right)=1 .
$$

As $m$ is large enough, we also have

$$
\varepsilon\left(\pi \times \chi_{m}, \frac{1}{2}\right)=-1
$$

by $[1,2.4 .12(3)]$, hence

$$
r_{\text {an }}\left(K, g, \chi_{m}\right) \equiv 1 \equiv h_{f}^{1}\left(K, V \otimes \chi_{m}\right)(\bmod 2) .
$$

Finally, as the restrictions of $\chi$ and $\chi_{m}$ to the torsion subgroup of $G^{(c)}$ coincide, we have

$$
r_{\text {an }}(K, g, \chi)-h_{f}^{1}(K, V \otimes \chi) \equiv r_{\text {an }}\left(K, g, \chi_{m}\right)-h_{f}^{1}\left(K, V \otimes \chi_{m}\right) \equiv 0(\bmod 2),
$$

by Proposition 2.1.2(5) below (which was earlier proved in $[13,12.6 .4 .7(v)]$ in the special case when $(c,(p))=(1))$. This finishes the proof of Theorem 0.4. 
1.5.2. - In fact, it is enough to appeal to a weaker non-vanishing result [1, Thm. 2.5.1], which does not require the CM points $x_{m}$ to be good and which yields, for $m$ large enough, a character $\chi_{m}: G^{(c)} \longrightarrow \overline{\mathbb{Q}}^{*}$ satisfying $1.5 .1(1)$ and 1.5.1(3), but not necessarily 1.5.1(2). The equality (1.5.1.5) in this case follows from [1, Prop. 2.6.2(2)].

\section{Iwasawa theory}

Throughout this section we assume that $g \in S_{k}(\mathfrak{n}(g), 1)$ is a potentially $p$-ordinary Hilbert modular newform of level $\mathfrak{n}(g)$; let $\pi=\pi(g)$. We also fix $K$ as in 0.1 and put $\eta=\eta_{K / F}$. Fix a non-empty subset $\Sigma=\left\{P_{1}, \ldots, P_{s}\right\} \subseteq \Sigma_{p}$ and an ideal $c \subset O_{F}$ relatively prime to $P_{1} \cdots P_{s}$. As in $[1,1.2 .2]$, set $K\left[c P^{\infty}\right]=\bigcup_{m \geq 1} K\left[c P_{1}^{m} \cdots P_{s}^{m}\right], G^{(c)}=\operatorname{Gal}\left(K\left[c P^{\infty}\right] / K\right)$ and $G_{0}^{(c)}=\left(G^{(c)}\right)_{\text {tors }}$.

\section{1. $\varepsilon$-factors}

2.1.1. - For a character $\chi: G^{(c)} \longrightarrow \mathbb{C}^{*}$ of finite order, let

$$
\varepsilon_{v}=\varepsilon\left(\pi_{v} \times \chi_{v}, \frac{1}{2}\right) \in\{ \pm 1\}, \quad \widetilde{\varepsilon}_{v}=\widetilde{\varepsilon}\left(\pi_{v} \times \chi_{v}, \frac{1}{2}\right) \in\{ \pm 1\},
$$

where

$$
\widetilde{\varepsilon}_{v}=\varepsilon_{v} \times \begin{cases}-1, & v \in \Sigma_{\chi}(g) \\ 1, & v \notin \Sigma_{\chi}(g)\end{cases}
$$

is the modified local $\varepsilon$-factor defined in $[13,12.6 .3 .10]$ (if $k>2$, then $\Sigma_{\chi}(g)=\varnothing$ and $\widetilde{\varepsilon}_{v}=\varepsilon_{v}$ for all $v$ ). If $\chi^{\prime}: G^{(c)} \longrightarrow \mathbb{C}^{*}$ is another character of finite order, denote the corresponding local $\varepsilon$-factors by $\varepsilon_{v}^{\prime}$ and $\widetilde{\varepsilon}_{v}^{\prime}$.

2.1.2. Proposition. - Let $\chi, \chi^{\prime}: G^{(c)} \longrightarrow \mathbb{C}^{*}$ be characters of finite order such that $\left.\chi\right|_{G_{0}^{(c)}}=$ $\left.\chi^{\prime}\right|_{G_{0}^{(c)}}$. If $v$ is a prime of $F$, then:

1. If $v \mid \infty$ or if $v \nmid \infty$ splits in $K / F$, then $\varepsilon_{v}=\varepsilon_{v}^{\prime}=\widetilde{\varepsilon}_{v}=\widetilde{\varepsilon}_{v}=1$.

2. If $v=P_{i}(i=1, \ldots, s)$, then $\widetilde{\varepsilon}_{v}=\widetilde{\varepsilon}_{v}=\eta_{v}(-1)$.

3. If $v \mid c d_{K / F}, v \neq P_{1}, \ldots, P_{s}$, then $\varepsilon_{v}=\varepsilon_{v}^{\prime}$ and $\widetilde{\varepsilon}_{v}=\widetilde{\varepsilon}_{v}$.

4. If $v \nmid \infty c d_{K / F}$, then $\varepsilon_{v}=\varepsilon_{v}^{\prime}$ (more precisely, $\chi_{v}=\chi_{v}^{\prime}=1$ if $v$ does not split in $K / F$ ) and

$$
\widetilde{\varepsilon}_{v}=\widetilde{\varepsilon}_{v}= \begin{cases}1, & \text { if } \pi_{v} \text { is not supercuspidal } \\ \eta_{v}(v)^{o\left(\pi_{v}\right)}, & \text { if } \pi_{v} \text { is supercuspidal. }\end{cases}
$$

5. $r_{\mathrm{an}}(K, g, \chi)-h_{f}^{1}(K, V \otimes \chi) \equiv r_{\mathrm{an}}\left(K, g, \chi^{\prime}\right)-h_{f}^{1}\left(K, V \otimes \chi^{\prime}\right)(\bmod 2)$.

Proof. - (1) [13, 12.6.2.2.1, 12.6.2.4].

(2) As $v \mid p$ and $g$ is potentially $p$-ordinary, $\pi_{v}$ is not supercuspidal; apply [13, 12.6.2.4(i), (ii)].

(3) Let $S=\left\{v \mid c d_{K / F}, v \neq P_{1}, \ldots, P_{s}\right\}$. By [1, 1.2.7], $G_{0}^{(c)}$ has a subgroup $G_{1}^{(c)} \stackrel{\sim}{\longrightarrow}$ $U^{\prime}\left(c, P_{1} \cdots P_{s}\right)=\prod_{v \in S} U_{v}^{\prime}$, where each $U_{v}^{\prime}$ is isomorphic to a quotient of $\left(K_{v}^{*}\right)^{\circ} / F_{v}^{*}$, via the reciprocity map $\operatorname{rec}_{K}$. If $v \in S$ does not split in $K / F$, then $\left(K_{v}^{*}\right)^{\circ}=K_{v}^{*}$, which means that $\chi_{v}$ depends only on $\left.\chi\right|_{G_{1}^{(c)}}$; thus $\chi_{v}=\chi_{v}^{\prime}$, which implies that $\varepsilon_{v}=\varepsilon_{v}^{\prime}$ and $\widetilde{\varepsilon}_{v}=\widetilde{\varepsilon}_{v}$. If $v \in S$ splits in $K / F$, we conclude by (1). 
(4) If $\pi_{v}$ is not supercuspidal, then $\widetilde{\varepsilon}_{v}=\widetilde{\varepsilon}_{v}=\eta_{v}(-1)=1$ (recall that $v$ is unramified in $K / F$, by assumption), by [13, 12.6.2.4(i), (ii)]. If $\pi_{v}$ is supercuspidal, then $\varepsilon_{v}=\widetilde{\varepsilon}_{v}=\varepsilon_{v}^{\prime}=$ $\widetilde{\varepsilon}_{v}=\eta_{v}(v)^{o\left(\pi_{v}\right)}$, by [13, 12.6.2.4(iii)]. If $v$ is inert in $K / F$, then $\chi_{v}=\chi_{v}^{\prime}=1$, as both $\chi_{v}$ and $\chi_{v}^{\prime}$ are unramified and $K_{v}^{*}=O_{K, v}^{*} F_{v}^{*}$. If $v$ splits in $K / F$, then $\varepsilon_{v}=\varepsilon_{v}^{\prime}$, by (1).

(5) As $\widetilde{\varepsilon}:=\prod_{v} \widetilde{\varepsilon}_{v}$ is equal to $\widetilde{\varepsilon}:=\prod_{v} \widetilde{\varepsilon}_{v}^{\prime}$, by (1)-(4), we deduce from [13, 12.6.4.3] that

$$
\begin{aligned}
\tilde{h}_{f}^{1}(K, V \otimes \chi)-h_{f}^{1}(K, V \otimes \chi) & r_{\mathrm{an}}(K, g, \chi) \equiv \\
& \tilde{h}_{f}^{1}\left(K, V \otimes \chi^{\prime}\right)-h_{f}^{1}\left(K, V \otimes \chi^{\prime}\right)+r_{\mathrm{an}}\left(K, g, \chi^{\prime}\right)(\bmod 2),
\end{aligned}
$$

where $\widetilde{h}_{f}^{1}(K, V \otimes \chi)$ denotes the dimension of the "extended Selmer group" $\widetilde{H}_{f}^{1}(K, V \otimes \chi)$ defined in $[13,12.5 .9 .2]$. On the other hand, [13, Thm. 10.7.17] implies that

$$
\widetilde{h}_{f}^{1}(K, V \otimes \chi) \equiv \widetilde{h}_{f}^{1}\left(K, V \otimes \chi^{\prime}\right)(\bmod 2),
$$

as explained in the proof of $[13,12.6 .4 .7(v)]$. Combining the two congruences, we obtain the desired result.

2.1.3. Proposition-Definition. - (1) $\varepsilon_{\lim }\left(\pi \times \chi_{0}, \frac{1}{2}\right):=\varepsilon\left(\pi \times \chi, \frac{1}{2}\right) \prod_{i=1}^{s} \eta_{P_{i}}(-1) \varepsilon_{P_{i}} \in\{ \pm 1\}$ depends only on $\pi$ and $\chi_{0}=\left.\chi\right|_{G_{0}^{(c)}}$.

(2) If $k>2$, then $\varepsilon_{\lim }\left(\pi \times \chi_{0}, \frac{1}{2}\right)=\varepsilon\left(\pi \times \chi, \frac{1}{2}\right)$ for any $\chi$ satisfying $\left.\chi\right|_{G_{0}^{(c)}}=\chi_{0}$.

(3) If $n \in \mathbb{N}$ is large enough and $\left(P_{1} \cdots P_{s}\right)^{n} \mid c(\chi)$, then $\varepsilon\left(\pi \times \chi, \frac{1}{2}\right)=\varepsilon_{\lim }\left(\pi \times \chi_{0}, \frac{1}{2}\right)$.

Proof. - (1) By Proposition 2.1.2, the individual terms in

$$
\varepsilon\left(\pi \times \chi, \frac{1}{2}\right) \prod_{i=1}^{s} \eta_{P_{i}}(-1) \varepsilon_{P_{i}}=\prod_{v \nmid P_{1} \cdots P_{s}} \eta_{v}(-1) \varepsilon_{v}
$$

are the same for $\chi$ and $\chi^{\prime}$.

(2) If $k>2$, then each $\pi_{P_{i}}$ is a principal series representation [15, 12.5.4], hence $\eta_{P_{i}}(-1) \varepsilon_{P_{i}}=1([15,12.6 .2 .4(\mathrm{i})])$.

(3) If $n$ is large enough, then each term $\eta_{P_{i}}(-1) \varepsilon_{P_{i}}$ is equal to 1 , by [11, Prop. 3.8] and [10, Thm. 20.6].

\subsection{Ranks}

2.2.1. - Fix a character $\chi_{0}: G_{0}^{(c)} \longrightarrow O_{L}^{*}$, where $L$ is a number field such that $i_{\infty}(L)$ contains all Hecke eigenvalues of $g$. Let $\mathfrak{p} \mid p$ be as in 0.1 ; put $O=O_{L, \mathfrak{p}}$, and $\Lambda=O\left[\left[G^{(c)}\right]\right]$. For any $O\left[G_{0}^{(c)}\right]$-module $M$, define

$$
M_{\left(\chi_{0}\right)}:=M \otimes_{O\left[G_{0}^{(c)}\right], \chi_{0}} O
$$

then

$$
\Lambda_{\left(\chi_{0}\right)} \stackrel{\sim}{\longrightarrow} O\left[\left[G^{(c)} / G_{0}^{(c)}\right]\right] \stackrel{\sim}{\longrightarrow} O\left[\left[X_{1}, \ldots, X_{r}\right]\right], \quad r=\sum_{i=1}^{s}\left[F_{P_{i}}: \mathbb{Q}_{p}\right]
$$


2.2.2. - We say that a prime $v$ of $F$ is exceptional if $v \mid p, v \nmid P_{1} \cdots P_{s}$ and $\pi_{v}=\mathrm{St} \otimes \mu$ $\left(\mu^{2}=1\right)$. Denote by $\mathcal{M}\left(g, K ; c, P_{1}, \ldots, P_{s} ; \chi_{0}\right)$ the set of all exceptional primes of $F$ that do not split in $K / F$ and satisfy

$$
\begin{cases}\left(\chi_{0}\right)_{w}=\mu \circ N_{K_{w} / F_{v}}, & \text { if } v \mid c d_{K / F} \\ \operatorname{ord}_{v}(\mathfrak{n}(g))=1, & \text { if } v \nmid c d_{K / F}\end{cases}
$$

(where $w$ is the unique prime of $K$ above $v$ ). If $k>2$, then there are no exceptional primes.

2.2.3. - Let $\chi: G^{(c)} \longrightarrow O^{\prime *}$ be a character of finite order, where $O^{\prime}$ is the ring of integers in a finite extension of $L_{\mathfrak{p}}$. The extended Selmer group of $V \otimes \chi([13,12.5 .9 .2])$ sits in an exact sequence

$$
0 \longrightarrow \bigoplus_{w|v| p} H^{0}\left(K_{w}, V_{v}^{-} \otimes \chi_{w}\right) \longrightarrow \widetilde{H}_{f}^{1}(K, V \otimes \chi) \longrightarrow H_{f}^{1}(K, V \otimes \chi) \longrightarrow 0,
$$

where $v($ resp., $w)$ is a prime of $F$ (resp., of $K$ ) and

$$
0 \longrightarrow V_{v}^{+} \longrightarrow V \longrightarrow V_{v}^{-} \longrightarrow 0
$$

is an exact sequence of $L_{\mathfrak{p}}\left[\operatorname{Gal}\left(\bar{F}_{v} / F_{v}\right)\right]$-modules arising from the potential $p$-ordinarity of $g$. Denote by $h^{0}, \widetilde{h}_{f}^{1}$ and $h_{f}^{1}$ the dimensions of the respective cohomology groups over the fraction field of $O^{\prime}$; then

$$
\widetilde{h}_{f}^{1}(K, V \otimes \chi)-h_{f}^{1}(K, V \otimes \chi)=\sum_{w|v| p} h^{0}\left(K_{w}, V_{v}^{-} \otimes \chi_{w}\right),
$$

where

$$
h^{0}\left(K_{w}, V_{v}^{-} \otimes \chi_{w}\right)= \begin{cases}1, & \pi_{v}=\mathrm{St} \otimes \mu\left(\mu^{2}=1\right), \chi_{w}=\mu \circ N_{K_{w} / F_{v}} \\ 0, & \text { otherwise }\end{cases}
$$

$([13,12.5 .8])$. In particular, $h^{0}\left(K_{w}, V_{v}^{-} \otimes \chi_{w}\right)=0$ and $\widetilde{h}_{f}^{1}(K, V \otimes \chi)=h_{f}^{1}(K, V \otimes \chi)$ if $k>2$.

2.2.4. Proposition. - Assume that $k=2$. Let $\chi: G^{(c)} \longrightarrow O^{* *}$ be a character of finite order satisfying $\left.\chi\right|_{G_{0}^{(c)}}=\chi_{0}$ and such that $\left(P_{1} \cdots P_{s}\right)^{n} \mid c(\chi)$, for large enough $n \in \mathbb{N}$. Then:

1. $\widetilde{h}_{f}^{1}(K, V \otimes \chi)-h_{f}^{1}(K, V \otimes \chi) \equiv\left|\mathcal{M}\left(g, K ; c, P_{1}, \ldots, P_{s} ; \chi_{0}\right)\right|(\bmod 2)$.

2. If there exists an exceptional prime of $F$ that splits in $K / F$, assume, in addition, that there is $i \in\{1, \ldots, s\}$ such that $F_{P_{i}}=\mathbb{Q}_{p}$. Then

$$
\widetilde{h}_{f}^{1}(K, V \otimes \chi)-h_{f}^{1}(K, V \otimes \chi)=\left|\mathcal{M}\left(g, K ; c, P_{1}, \ldots, P_{s} ; \chi_{0}\right)\right| .
$$

Proof. - If $w$ is a prime of $K$ dividing $P_{1} \cdots P_{s}$, then $\chi_{w}^{2} \neq 1$ if $n$ is large enough, which means that $w$ does not contribute to the R.H.S. of (2.2.3.1).

Let $v$ be an exceptional prime of $F$. If $v$ does not split in $K / F$ and $v \mid c d_{K / F}$, then $\chi_{w}=$ $\left(\chi_{0}\right)_{w}$ (note that the decomposition group of $v$ in $G^{(c)}$ is finite, as $v$ does not split in $K / F$ ) and

$$
h^{0}\left(K_{w}, V_{v}^{-} \otimes \chi_{w}\right)=h^{0}\left(K_{w}, V_{v}^{-} \otimes\left(\chi_{0}\right)_{w}\right)= \begin{cases}1, & v \in \mathcal{M}\left(g, K ; c, P_{1}, \ldots, P_{s} ; \chi_{0}\right) \\ 0, & v \notin \mathcal{M}\left(g, K ; c, P_{1}, \ldots, P_{s} ; \chi_{0}\right) .\end{cases}
$$


If $v$ does not split in $K / F$ and $v \nmid c d_{K / F}$, then $v$ is inert in $K / F\left(v O_{K}=w\right)$ and $\chi_{w}$ is unramified; thus $\chi_{w}=1$ and (using [13, (12.3.9.1)])

$$
\begin{aligned}
h^{0}\left(K_{w}, V_{v}^{-} \otimes \chi_{w}\right) & = \begin{cases}1, & \mu \text { is unramified } \Longleftrightarrow \operatorname{ord}_{v}(\mathfrak{n}(g))=1 \\
0, & \text { otherwise }\end{cases} \\
& = \begin{cases}1, & v \in \mathcal{M}\left(g, K ; c, P_{1}, \ldots, P_{s} ; \chi_{0}\right) \\
0, & v \notin \mathcal{M}\left(g, K ; c, P_{1}, \ldots, P_{s} ; \chi_{0}\right) .\end{cases}
\end{aligned}
$$

If $v$ splits in $K / F\left(v O_{K}=w w^{\prime}\right)$, then the $h^{0}$-terms in (2.2.3.1) for $w$ and $w^{\prime}$ are the same; this proves (1). Under the assumptions of (2), there is $i$ such that $\operatorname{Gal}\left(K\left[P_{i}^{\infty}\right] / K\right)$ is isomorphic to a product of $\mathbb{Z}_{p}$ by a finite abelian group. As the decomposition groups of $w$ and $w^{\prime}$ in $\operatorname{Gal}\left(K\left[P_{i}^{\infty}\right] / K\right)$ are infinite, it follows that $\chi_{w}^{2}, \chi_{w^{\prime}}^{2} \neq 1$ if $n$ is large enough; thus $w$ and $w^{\prime}$ do not contribute to the R.H.S. of (2.2.3.1).

2.2.5. Proposition. - Fix a $G_{F}$-stable O-lattice $T \subset V$. Let $w|v| p$ be as in 2.2.3. Define

$$
Z_{w}=\underset{\alpha}{\lim } \bigoplus_{w_{\alpha} \mid w} H^{0}\left(\left(K_{\alpha}\right)_{w_{\alpha}},(V / T)_{v}^{-}\right),
$$

where $K_{\alpha} / K$ runs through all finite subextensions of $K\left[c P_{1}^{\infty} \cdots P_{s}^{\infty}\right] / K$ and $(V / T)_{v}^{-}$is the image of $V_{v}^{-}$in $V / T$. Then

$$
\operatorname{cork}_{\Lambda_{\left(\chi_{0}\right)}} \bigoplus_{w \mid v}\left(Z_{w}\right)_{\left(\chi_{0}\right)}= \begin{cases}1, & v \in \mathcal{M}\left(g, K ; c, P_{1}, \ldots, P_{s} ; \chi_{0}\right) \\ 0, & v \notin \mathcal{M}\left(g, K ; c, P_{1}, \ldots, P_{s} ; \chi_{0}\right) .\end{cases}
$$

Proof. - If the decomposition group of $w$ in $K\left[c P_{1}^{\infty} \cdots P_{s}^{\infty}\right] / K$ is infinite, then the L.H.S. is equal to zero $([13,12.6 .4 .10])$. We can assume, therefore, that $v$ is exceptional and does not split in $K / F$. Choose an isomorphism $G^{(c)} \stackrel{\sim}{\longrightarrow} G_{0}^{(c)} \times\left(G^{(c)} / G_{0}^{(c)}\right)$; then $\chi_{0}$ becomes a character of $G^{(c)}$ and $D\left(\left(Z_{w}\right)_{\left(\chi_{0}\right)}\right)=D\left(H^{0}\left(K_{w},(V / T)_{v}^{-} \otimes\left(\chi_{0}^{-1}\right)_{w}\right)\right) \otimes_{O} \Lambda_{\left(\chi_{0}\right)}$, where $D(-)$ denotes the Pontrjagin dual (cf. $[13,9.6 .5])$; thus

$$
\operatorname{cork}_{\Lambda_{\left(\chi_{0}\right)}}\left(Z_{w}\right)_{\left(\chi_{0}\right)}=h^{0}\left(K_{w}, V_{v}^{-} \otimes\left(\chi_{0}^{-1}\right)_{w}\right)= \begin{cases}1, & v \in \mathcal{M}\left(g, K ; c, P_{1}, \ldots, P_{s} ; \chi_{0}\right) \\ 0, & v \notin \mathcal{M}\left(g, K ; c, P_{1}, \ldots, P_{s} ; \chi_{0}\right),\end{cases}
$$

by the proof of Proposition 2.2.4.

2.2.6. - Fix a finite set $S$ of primes of $F$ containing $\Sigma_{p} \cup \Sigma_{\infty}$ and all primes dividing $\mathfrak{n}(g)$, and a subset $\Sigma^{\prime} \subseteq S-\left(\Sigma_{p} \cup \Sigma_{\infty}\right)$. As in [13, 12.5.9.1], consider the corresponding Greenberg's local conditions for $T$ and $V / T$. In order to simplify the notation, put $K_{\infty}=K\left[c P_{1}^{\infty} \cdots P_{s}^{\infty}\right]$.

2.2.7. Proposition. - (1) The (co)-ranks

$$
\forall j=1,2 \quad \operatorname{rk}_{\Lambda_{\left(\chi_{0}\right)}} \widetilde{H}_{f, \text { Iw }}^{j}\left(K_{\infty} / K, T\right)_{\left(\chi_{0}^{ \pm 1}\right)}=\operatorname{cork}_{\Lambda_{\left(\chi_{0}\right)}} \widetilde{H}_{f}^{j}\left(K_{S} / K_{\infty}, V / T\right)_{\left(\chi_{0}^{ \pm 1}\right)}=r(g)
$$

(where $\widetilde{H}_{f, \text { Iw }}^{j}\left(K_{\infty} / K, T\right)$ is the $\Lambda$-module defined in $\left.[13,8.8 .5]\right)$ are equal to the same integer $r(g)$ and do not depend on $S$ and $\Sigma^{\prime}$.

(2) Assume that $\varepsilon_{\lim }\left(\pi \times \chi_{0}, \frac{1}{2}\right)=-1$. If $g$ has $C M$ by a totally imaginary quadratic extension $K^{\prime}$ of $F$, assume that $p \neq 2$ and $K^{\prime} \not \subset K_{\infty}^{\operatorname{Ker}\left(\chi_{0}\right)}$. Then

$$
r(g) \equiv 1+\left|\mathcal{M}\left(g, K ; c, P_{1}, \ldots, P_{s} ; \chi_{0}\right)\right|(\bmod 2) .
$$


Proof. - (1) See the proof of [13, 12.6.4.12].

(2) Let $\chi: G^{(c)} \longrightarrow O^{\prime *}$ be a character of finite order such that $\left.\chi\right|_{G_{0}^{(c)}}=\chi_{0}$ and $\left(P_{1} \cdots P_{s}\right)^{n} \mid c(\chi)$, for large enough $n \in \mathbb{N}$. We have

$$
\begin{aligned}
r(g) & \equiv \widetilde{h}_{f}^{1}(K, V \otimes \chi)(\bmod 2) & & {[13,10.7 .17] } \\
& \equiv h_{f}^{1}(K, V \otimes \chi)+\left|\mathcal{M}\left(g, K ; c, P_{1}, \ldots, P_{s} ; \chi_{0}\right)\right|(\bmod 2) & & \text { Proposition 2.2.4(1) } \\
& \equiv 1+\left|\mathcal{M}\left(g, K ; c, P_{1}, \ldots, P_{s} ; \chi_{0}\right)\right|(\bmod 2) & & \text { Theorem 0.4(1) }
\end{aligned}
$$

(Theorem 0.4(1) applies, as $\varepsilon\left(\pi \times \chi, \frac{1}{2}\right)=\varepsilon_{\lim }\left(\pi \times \chi_{0}, \frac{1}{2}\right)=-1$, by Proposition-Definition 2.1.3(3)).

2.2.8. Proposition. - Assume that $k=2$ and $\varepsilon_{\lim }\left(\pi \times \chi_{0}, \frac{1}{2}\right)=-1$. If $g$ has CM by a totally imaginary quadratic extension $K^{\prime}$ of $F$, assume that $p \neq 2$ and $K^{\prime} \not \subset K_{\infty}^{\operatorname{Ker}\left(\chi_{0}\right)}$. If there exists an exceptional prime of $F$ that splits in $K / F$, assume, in addition, that there is $i \in\left\{P_{1}, \ldots, P_{s}\right\}$ such that $F_{P_{i}}=\mathbb{Q}_{p}$. Then:

(1) The (co)-ranks in Proposition 2.2.7(1) are equal to

$$
r(g)=1+\left|\mathcal{M}\left(g, K ; c, P_{1}, \ldots, P_{s} ; \chi_{0}\right)\right| .
$$

(2) $\operatorname{cork}_{\Lambda_{\left(\chi_{0}\right)}} S_{V / T}^{\mathrm{str}}\left(K_{\infty}\right)_{\left(\chi_{0}^{ \pm 1}\right)}=1$, where $S_{V / T}^{\mathrm{str}}\left(K_{\infty}\right) \subset H^{1}\left(K_{\infty}, V / T\right)$ is Greenberg's strict Selmer group [13, 9.6.5].

(3) If $K \subset K_{\infty}^{\prime} \subset K_{\infty}$ is an intermediate field such that $\chi_{0}$ factors through $\chi_{0}^{\prime}$ : $\operatorname{Gal}\left(K_{\infty}^{\prime} / K\right)_{\text {tors }} \longrightarrow O_{L}^{*}$, then the (co)-ranks

$\forall j=1,2 \quad \operatorname{rk}_{\Lambda_{\left(\chi_{0}^{\prime}\right)}^{\prime}} \widetilde{H}_{f, \mathrm{Iw}}^{j}\left(K_{\infty}^{\prime} / K, T\right)_{\left(\chi_{0}^{\prime \pm 1}\right)}=\operatorname{cork}_{\Lambda_{\left(\chi_{0}^{\prime}\right)}^{\prime}} \widetilde{H}_{f}^{j}\left(K_{S} / K_{\infty}^{\prime}, V / T\right)_{\left(\chi_{0}^{\prime \pm 1}\right)}=r^{\prime}(g)$ (where $\Lambda^{\prime}=O\left[\left[\operatorname{Gal}\left(K_{\infty}^{\prime} / K\right)\right]\right]$ ) are all equal, do not depend on $S$ and $\Sigma^{\prime}$, and satisfy

$$
r^{\prime}(g) \geq r(g), \quad r^{\prime}(g) \equiv r(g)(\bmod 2) .
$$

Proof (cf. $[13,12.6 .4 .12,12.9 .8])$. - (1), (2) Denote the coranks in (2) by $s_{ \pm}(g)$. The exact sequence

$$
\begin{aligned}
H^{0}\left(K_{\infty}, V / T\right)_{\left(\chi_{0}^{ \pm 1}\right)} \longrightarrow \bigoplus_{w|v| p}\left(Z_{w}\right)_{\left(\chi_{0}^{ \pm 1}\right)} \longrightarrow \widetilde{H}_{f}^{1}\left(K_{S} / K_{\infty}, V / T\right)_{\left(\chi_{0}^{ \pm 1}\right)} \longrightarrow & \\
& \longrightarrow S_{V / T}^{\operatorname{str}}\left(K_{\infty}\right)_{\left(\chi_{0}^{ \pm 1}\right)} \longrightarrow 0
\end{aligned}
$$

([13, (9.6.5.1)]) combined with Proposition 2.2.5 and 2.2.7 implies that

$$
r(g)=s_{ \pm}(g)+\left|\mathcal{M}\left(g, K ; c, P_{1}, \ldots, P_{s} ; \chi_{0}\right)\right| .
$$

For $m \in \mathbb{N}$ large enough, let $\chi_{m}$ be as in 1.5.1; then

$$
h^{1}\left(K, V \otimes \chi_{m}\right)=1, \quad \widetilde{h}^{1}\left(K, V \otimes \chi_{m}\right)=1+\left|\mathcal{M}\left(g, K ; c, P_{1}, \ldots, P_{s} ; \chi_{0}\right)\right|,
$$

by (1.5.1.4) and Proposition 2.2.4(2). Finally,

$$
\widetilde{h}^{1}\left(K, V \otimes \chi_{m}\right) \geq r(g), \quad \widetilde{h}^{1}\left(K, V \otimes \chi_{m}\right) \equiv r(g)(\bmod 2)
$$


by [13, 10.7.17], which implies that

$$
s_{ \pm}(g)=1, \quad r(g)=1+\left|\mathcal{M}\left(g, K ; c, P_{1}, \ldots, P_{s} ; \chi_{0}\right)\right| .
$$

(3) This follows from [13, 8.10.11(ii)] and [13, 10.7.17(iii)].

2.2.9. Proposition. - Let $R$ be the quotient of the ordinary Hecke algebra defined in [13, 12.7.7] $(\operatorname{Spec}(R)$ is the branch of the cyclotomic Hida family of parallel weight passing through the p-stabilisation $f^{0}$ of a p-ordinary twist of $g$ ). Let $\bar{R}=R\left[\left[G^{(c)}\right]\right]$ and $\overline{\mathfrak{q}}=\bar{R} \cdot \operatorname{Ker}\left(\chi_{0}: O\left[G_{0}^{(c)}\right] \longrightarrow O\right)(\overline{\mathfrak{q}}$ is a minimal prime of $\bar{R})$. Let $\mathcal{T}$ be the big Galois representation defined in $[13,12.7 .15 .3]$ ( then $\mathcal{T}_{\mathcal{P}} / \mathcal{P} \mathcal{T}_{\mathcal{P}} \stackrel{\sim}{\longrightarrow} V$, where $\mathcal{P}$ is the arithmetic point of $R$ corresponding to $\left.f^{0}\right)$. Assume that $\varepsilon_{\lim }\left(\pi \times \chi_{0}, \frac{1}{2}\right)=-1$. If $g$ has $C M$ by a totally imaginary quadratic extension $K^{\prime}$ of $F$, assume that $p \neq 2$ and $K^{\prime} \not \subset K_{\infty}^{\operatorname{Ker}\left(\chi_{0}\right)}$. Then:

(1) The ranks

$$
\forall j=1,2 \quad \operatorname{rk}_{\overline{R_{\bar{q}}}} \widetilde{H}_{f, \text { Iw }}^{j}\left(K_{\infty} / K, \mathcal{T}\right)_{\overline{\bar{q}}}=\operatorname{rk}_{\overline{R_{\bar{q}}}}\left(\widetilde{H}_{f, \text { Iw }}^{j}\left(K_{\infty} / K, \mathcal{T}\right)^{\iota}\right)_{\overline{\mathfrak{q}}}=1 .
$$

(2) For all but finitely many arithmetic points $\mathcal{P}^{\prime}$ of $R, r\left(g_{\mathcal{P}^{\prime}}\right)=1$, where $g_{\mathcal{P}^{\prime}} \in S_{k^{\prime}}\left(\mathfrak{n}^{\prime}, 1\right)$ satisfies $V\left(g_{\mathcal{P}^{\prime}}\right)\left(k^{\prime} / 2\right) \stackrel{\sim}{\longrightarrow} \mathcal{T}_{\mathcal{P}^{\prime}} / \mathcal{P}^{\prime} \mathcal{T}_{\mathcal{P}^{\prime}}$.

Proof (cf. [13, 12.9.8, 12.9.11]). - The four ranks in (1) are equal, by [13, 12.7.15.7(i), 12.7.16.8(i)]; denote their common value by $h$. Let $g^{\prime}=g_{\mathcal{P}^{\prime}}$ be as in (2). According to [13, 12.7.15.8(iii)],

$$
h \leq r\left(g^{\prime}\right), \quad h \equiv r\left(g^{\prime}\right)(\bmod 2) .
$$

Choose $\mathcal{P}^{\prime}$ such that $k^{\prime}=2$ and $\pi\left(g^{\prime}\right)_{v}$ is a principal series representation for all $v \mid p$ (cf. [13, 12.7.10]); then $\mathcal{M}\left(g, K ; c, P_{1}, \ldots, P_{s} ; \chi_{0}\right)=\varnothing$. Applying (a slight variant of) Proposition 2.2.8(1) to $g^{\prime}$, we obtain $r\left(g^{\prime}\right)=1$, hence $h=1$. The statement (2) follows from the fact that $h=r\left(g^{\prime}\right)$ for almost all $\mathcal{P}^{\prime}$.

\section{Proof of Theorem 0.8}

\subsection{Kummer theory}

3.1.1. - As in 0.7 , let $q \neq 2$ be a prime number and $F_{0}$ a totally real number field such that $F_{0} \cap \mathbb{Q}\left(\mu_{q^{\infty}}\right)=\mathbb{Q}$. Let $a \in O_{F_{0}}-\{0\}$ be an element satisfying $a \notin F_{0}^{* q}$ and, for each finite prime $v_{0}$ of $F_{0}$ not dividing $q$, ord $v_{0}(a)<q$. For $r \geq 1$, put $F_{r}=F_{0} \mathbb{Q}\left(\mu_{q^{r}}\right)^{+}, K_{r}=F_{0}\left(\mu_{q^{r}}\right)$.

3.1.2. - Let $r \geq 1$. As $F_{0} \cap \mathbb{Q}\left(\mu_{q^{\infty}}\right)=\mathbb{Q}$,

$$
\operatorname{Gal}\left(K_{r} / F_{0}\right) \stackrel{\sim}{\longrightarrow} \operatorname{Gal}\left(\mathbb{Q}\left(\mu_{q^{r}}\right) / \mathbb{Q}\right) \stackrel{\sim}{\longrightarrow}\left(\mathbb{Z} / q^{r} \mathbb{Z}\right)^{*} .
$$

The map

$$
F_{0}^{*} \otimes \mathbb{Z} / q^{r} \mathbb{Z}=H^{1}\left(F_{0}, \mu_{q^{r}}\right) \stackrel{\text { res }}{\longrightarrow} H^{1}\left(K_{r}, \mu_{q^{r}}\right)=K_{r}^{*} \otimes \mathbb{Z} / q^{r} \mathbb{Z}
$$

is injective, as

$$
|\operatorname{Ker}(\mathrm{res})|=\left|H^{1}\left(K_{r} / F_{0}, \mu_{q^{r}}\right)\right|=\left|\widehat{H}^{0}\left(K_{r} / F_{0}, \mu_{q^{r}}\right)\right| \leq\left|H^{0}\left(F_{0}, \mu_{q^{r}}\right)\right|=1 ;
$$

thus $a \notin K_{r}^{* q}$, which yields, by Kummer theory, an isomorphism

$$
\operatorname{Gal}\left(K_{r}\left(\sqrt[q^{r}]{a}\right) / K_{r}\right) \stackrel{\sim}{\longrightarrow} \mu_{q^{r}}, \quad g \mapsto g\left(\sqrt[q^{r}]{a}\right) / \sqrt[q^{r}]{a} .
$$


A choice of a compatible system of roots $\sqrt[q]{a} \in \overline{\mathbb{Q}}$ such that $\left(\sqrt[q^{r}]{a}\right)^{q}=\sqrt[q^{r-1}]{a}$ determines a compatible system of isomorphisms

$$
\operatorname{Gal}\left(K_{r}\left(\sqrt[q^{r}]{a}\right) / F_{0}\right) \stackrel{\sim}{\longrightarrow} \mu_{q^{r}} \rtimes\left(\mathbb{Z} / q^{r} \mathbb{Z}\right)^{*},
$$

under which $\operatorname{Gal}\left(K_{r}\left(\sqrt[q^{r}]{a}\right) / F_{0}\left(\sqrt[q^{r}]{a}\right)\right)$ corresponds to $\left(\mathbb{Z} / q^{r} \mathbb{Z}\right)^{*}$.

3.1.3. Proposition. - Let $H$ be a finite group acting on a finite abelian group $A$; let $G=A \rtimes H$. Let $L$ be a field of characteristic zero containing all roots of unity of order equal to the exponent of $A$. Then the $L[G]$-module $L[G / H]$ decomposes into simple $L[G]$-modules as follows:

$$
L[G / H]=\bigoplus_{\left[\chi: A \longrightarrow L^{*}\right]} \operatorname{Ind}_{A \rtimes H_{\chi}}^{A \rtimes H}(\chi \otimes 1)
$$

where $\chi$ runs through a set of representatives of $H \backslash \operatorname{Hom}\left(A, L^{*}\right)$ and $H_{\chi} \subset H$ is the stabiliser of $\chi$.

Proof. - Easy exercise.

3.1.4. - For a subfield $k \subset \overline{\mathbb{Q}}$, put $G_{k}=\operatorname{Gal}(\overline{\mathbb{Q}} / k)$. If $k^{\prime} / k$ is a finite subextension of $\overline{\mathbb{Q}} / k$, denote by

$$
\operatorname{Res}_{k^{\prime} / k}:\left(G_{k}-\operatorname{Mod}\right) \longrightarrow\left(G_{k^{\prime}}-\operatorname{Mod}\right), \quad \operatorname{Ind}_{k^{\prime} / k}:\left(G_{k^{\prime}}-\operatorname{Mod}\right) \longrightarrow\left(G_{k}-\operatorname{Mod}\right)
$$

the corresponding restriction and induction functors, respectively.

In the situation of 3.1.2, apply Proposition 3.1.3 to

$$
A=\operatorname{Gal}\left(K_{r}\left(\sqrt[q^{r}]{a}\right) / K_{r}\right) \stackrel{\sim}{\longrightarrow} \mu_{q^{r}}, \quad H=\operatorname{Gal}\left(K_{r}\left(\sqrt[q^{r}]{a}\right) / F_{0}\left(\sqrt[q^{r}]{a}\right)\right) \stackrel{\sim}{\longrightarrow}\left(\mathbb{Z} / q^{r} \mathbb{Z}\right)^{*} ;
$$

we obtain, for any field $L \supset \mathbb{Q}\left(\mu_{q^{r}}\right)$,

$$
\operatorname{Ind}_{F_{0}(\sqrt[a]{a}) / F_{0}}(\mathbb{Z}) \otimes L=L[A \rtimes H / H]=\bigoplus_{s=0}^{r} \rho_{s}, \quad \rho_{s}=\operatorname{Ind}_{K_{s} / F_{0}}\left(\chi_{s}\right),
$$

where $\chi_{s}: \operatorname{Gal}\left(K_{s}\left(\sqrt[q^{s}]{a}\right) / K_{s}\right) \hookrightarrow L^{*}$ is an injective character (of order $q^{s}$ ) and $\rho_{s}$ is an absolutely irreducible representation of $G$ of dimension $\varphi\left(q^{s}\right)$. If $s \geq 1$, denote by $\rho$ the nontrivial element of $\operatorname{Gal}\left(K_{s} / F_{s}\right) \stackrel{\sim}{\longrightarrow} \operatorname{Gal}\left(\mathbb{Q}\left(\mu_{q^{s}}\right) / \mathbb{Q}\left(\mu_{q^{s}}\right)^{+}\right)$; then ${ }^{\rho} \chi_{s}=\chi_{s}^{-1}$. As the order of $\chi_{s}$ is odd, it follows that $\chi_{s}$ (more precisely, $\chi_{s} \circ \operatorname{rec}_{K_{s}}$ ) factors through $\mathbb{A}_{K_{s}}^{*} / K_{s}^{*} \mathbb{A}_{F_{s}}^{*}$.

If $M$ is an $L\left[G_{F_{0}}\right]$-module, the projection formula

$$
\operatorname{Ind}_{k^{\prime} / k}\left(X \otimes_{L} \operatorname{Res}_{k^{\prime} / k}(Y)\right)=\operatorname{Ind}_{k^{\prime} / k}(X) \otimes_{L} Y
$$

together with (3.1.4.1) imply that

$$
\operatorname{Ind}_{F_{0}(\sqrt[r]{a}) / F_{0}} \circ \operatorname{Res}_{F_{0}(\sqrt[r]{a}) / F_{0}}(M)=\bigoplus_{s=0}^{r} \operatorname{Ind}_{K_{s} / F_{0}}\left(\operatorname{Res}_{K_{s} / F_{0}}(M) \otimes \chi_{s}\right) .
$$

\section{2. $\varepsilon$-factors}

3.2.1. - In the situation of 3.1.1, let $g_{0} \in S_{k}\left(\mathfrak{n}_{0}, 1\right)$ be a cuspidal Hilbert modular newform over $F_{0}$ of parallel weight $k$, trivial central character and level $\mathfrak{n}_{0}$; put $V=V_{\mathfrak{p}}\left(g_{0}\right)(k / 2)$. For $r \geq 1$, denote by $g_{r}$ the base change of $g_{0}$ over $F_{r}$ (i.e., $\pi\left(g_{r}\right)=B C_{F_{r} / F}\left(\pi\left(g_{0}\right)\right)$ ). 
3.2.2. - It follows from (3.1.4.2) and Shapiro's Lemma that

$$
\forall r \geq 1 \quad H_{f}^{1}\left(F_{0}\left(\sqrt[a^{r}]{a}\right), V\right)=H_{f}^{1}\left(F_{0}, V\right) \oplus \bigoplus_{s=1}^{r} H_{f}^{1}\left(K_{s}, V \otimes \chi_{s}\right)
$$

(taking $L \supset \mathbb{Q}\left(\mu_{q^{r}}\right)$ in 0.1 ), hence

$$
\forall r \geq 1 \quad h_{f}^{1}\left(F_{0}\left(\sqrt[q^{r}]{a}\right), V\right)-h_{f}^{1}\left(F_{0}\left(\sqrt[r^{r-1}]{a}\right), V\right)=h_{f}^{1}\left(K_{r}, V \otimes \chi_{r}\right) .
$$

3.2.3. Proposition. - Assume that $\left(\forall v_{0} \mid a\right) \pi\left(g_{0}\right)_{v_{0}}$ is not supercuspidal and that $\left(\forall v_{0} \mid q\right)$ $\pi\left(g_{0}\right)_{v_{0}}$ is a principal series representation. Then:

$$
\forall r \geq 1 \quad \varepsilon\left(\pi\left(g_{r}\right) \times \chi_{r}, \frac{1}{2}\right)=\left(\frac{d}{q}\right), \quad d=(-1)^{\left[F_{0}: \mathbb{Q}\right]} N\left(\mathfrak{n}_{0}^{(a q)}\right),
$$

where $\mathfrak{a}^{(\mathfrak{b})}=\mathfrak{a} /\left(\mathfrak{a}, \mathfrak{b}^{\infty}\right)$ is the prime-to-b part of $\mathfrak{a}$, for any non-zero ideals $\mathfrak{a}, \mathfrak{b}$ in the ring of integers of a given number field.

Proof. - Fix $r \geq 1$. Let $v$ be a finite prime of $F_{r}$. Our assumptions imply that

$$
\begin{gathered}
v \text { ramifies in } K_{r} / F_{r} \Longleftrightarrow v \mid q \\
v|a, v \nmid q \Longrightarrow v| c\left(\chi_{r}\right) \Longrightarrow v \mid a q .
\end{gathered}
$$

It follows that the condition $H\left(K_{r}, \chi_{r}\right)$ from [13, 12.6.3.5] is satisfied. Applying [13, 12.6.3.9], we obtain

$$
\varepsilon\left(\pi\left(g_{r}\right) \times \chi_{r}, \frac{1}{2}\right)=(-1)^{\left[F_{r}: \mathbb{Q}\right]} \eta_{K_{r} / F_{r}}\left(\mathfrak{n}\left(g_{r}\right)^{(q)}\right)(-1)^{\left|R(1)^{0} \cap\left\{v \nmid c\left(\chi_{r}\right)\right\}\right|}(-1)^{\left|R(1)^{-} \cap\left\{v \mid c\left(\chi_{r}\right)\right\}\right|},
$$

where $\mathfrak{n}\left(g_{r}\right) \subset O_{F_{r}}$ is the level of $g_{r}$ and $R(1)^{0}$ (resp., $R(1)^{-}$) contains those elements of $\Sigma_{1}\left(g_{r}\right)$ which are ramified (resp., inert) in $K_{r} / F_{r}$ (see [13, 12.6.3.2]). By (3.2.3.1), we have $R(1)^{0}=\varnothing$ and

$$
\begin{aligned}
R(1)^{-} & \cap\left\{v \mid c\left(\chi_{r}\right)\right\} \\
& =\left\{v \mid a ; v \text { is inert in } K_{r} / F_{r}, \pi\left(g_{r}\right)=\mathrm{St} \otimes \mu, \mu: F_{r, v}^{*} \longrightarrow\{ \pm 1\} \text { is unramified }\right\} \\
& =\left\{v \mid a ; v \text { is inert in } K_{r} / F_{r}, \pi\left(g_{r}\right)=\mathrm{St} \otimes \mu, \mu: F_{r, v}^{*} \longrightarrow\{ \pm 1\}, o\left(\pi\left(g_{r}\right)_{v}\right)=1\right\} \\
& =\left\{v \mid a ; v \text { is inert in } K_{r} / F_{r}, \pi\left(g_{r}\right)=\mathrm{St} \otimes \mu, \mu: F_{r, v}^{*} \longrightarrow\{ \pm 1\}, 2 \nmid o\left(\pi\left(g_{r}\right)_{v}\right)\right\}
\end{aligned}
$$

(as $\pi\left(g_{r}\right)_{v}$ is not supercuspidal), hence

$$
\varepsilon\left(\pi\left(g_{r}\right) \times \chi_{r}, \frac{1}{2}\right)=(-1)^{\left[F_{r}: \mathbb{Q}\right]} \eta_{K_{r} / F_{r}}\left(\mathfrak{n}\left(g_{r}\right)^{(a q)}\right) .
$$

As $F_{r} / F$ is unramified outside $q$, it follows that

$$
\mathfrak{n}\left(g_{r}\right)^{(q)}=\mathfrak{n}_{0}^{(q)} O_{F_{r}}, \quad \mathfrak{n}\left(g_{r}\right)^{(a q)}=\mathfrak{n}_{0}^{(a q)} O_{F_{r}} .
$$

Let $F^{\prime}=\mathbb{Q}\left(\mu_{q^{r}}\right)^{+}, K^{\prime}=\mathbb{Q}\left(\mu_{q^{r}}\right)$. If $I_{0} \subset O_{F_{0}}$ is an ideal prime to $q$, let $I_{r}=I_{0} O_{F_{r}}$; then

$$
\begin{aligned}
\eta_{K_{r} / F_{r}}\left(I_{r}\right)=\eta_{K^{\prime} / F^{\prime}}\left(N_{F_{r} / F^{\prime}}\left(I_{r}\right)\right) & \\
& \equiv N\left(I_{r}\right)\left(\bmod q^{r}\right) \equiv N\left(I_{0}\right)^{\varphi\left(q^{r}\right) / 2} \equiv N\left(I_{0}\right)^{(q-1) / 2}(\bmod q),
\end{aligned}
$$

hence

$$
\eta_{K_{r} / F_{r}}\left(I_{0} O_{F_{r}}\right)=\left(\frac{N\left(I_{0}\right)}{q}\right), \quad(-1)^{\left[F_{r}: \mathbb{Q}\right]} \eta_{K_{r} / F_{r}}\left(I_{0} O_{F_{r}}\right)=\left(\frac{(-1)^{\left[F_{0}: \mathbb{Q}\right]} N\left(I_{0}\right)}{q}\right) .
$$

Taking $I_{0}=\mathfrak{n}_{0}^{(a q)}$, we obtain the desired result. 
3.2.4. - The proof of Proposition 3.2.3 shows that the formula for $\varepsilon\left(\pi\left(g_{r}\right) \times \chi_{r}, \frac{1}{2}\right)$ still holds if we replace the assumption " $\left.\forall v_{0} \mid q\right) \pi\left(g_{0}\right)_{v_{0}}$ is a principal series representation" by " $\left(\forall v_{0} \mid q\right) \pi\left(g_{0}\right)_{v_{0}}$ is not supercuspidal, and the extension $K_{r}\left(\sqrt[a^{r}]{a}\right) / K_{r}$ is ramified at all primes above $q$ ", as $R(1)^{0} \cap\left\{v \nmid c\left(\chi_{r}\right)\right\}=\varnothing$ in this case.

\subsection{End of the proof}

3.3.1. - The statement of Theorem 0.8(1) follows from Proposition 3.2.3.

3.3.2. - By (3.2.2.2), the first line in the statement of Theorem 0.8(2) will follow from Theorem 0.4(1) applied to each $g_{s}$ and $\chi_{s}(1 \leq s \leq r)$. As $g_{0}$ is potentially $p$-ordinary, so is $g_{s}$. By 3.3.1, $\nmid r_{\mathrm{an}}\left(K_{s}, g_{s}, \chi_{s}\right)$, so it remains to check that if $g_{s}$ has CM by a totally imaginary quadratic extension $K^{\prime}$ of $F_{s}$, then $K^{\prime}$ is not contained in $K_{s}\left(\sqrt[q^{s}]{a}\right)$. If $K^{\prime} \subset K_{s}\left(\sqrt[q^{s}]{a}\right)$, then $K^{\prime}=K_{s}$ and $g_{0}$ has $\mathrm{CM}$ by a totally imaginary quadratic extension $K_{0}^{\prime}$ of $F_{0}$ such that $F_{r} K_{0}^{\prime}=K_{s}$. However, the only quadratic extension of $F_{0}$ contained in $K_{s}$ is $F_{0}\left(\sqrt{q^{*}}\right)$, where $q^{*}=(-1)^{(q-1) / 2} q$; thus $q \equiv 3(\bmod 4)$ and $K_{0}^{\prime}=F_{0}(\sqrt{-q})$, which contradicts the assumptions.

It remains to show that, in the case when $2 \nmid\left[F_{0}: \mathbb{Q}\right], g_{0}$ cannot have CM by $K_{0}^{\prime}=$ $F_{0}(\sqrt{-q}), q \equiv 3(\bmod 4)$. If that were the case, then each finite prime $v_{0}$ of $F_{0}$ ramified in $K_{0}^{\prime} / F_{0}$ would divide $q$, hence $\pi\left(g_{0}\right)_{v_{0}}$ would not be supercuspidal; thus $\pi\left(g_{0}\right)_{v_{0}}=\pi\left(\mu, \mu \eta_{v_{0}}^{\prime}\right)$, where $\eta^{\prime}=\eta_{K_{0}^{\prime} / F_{0}}([13,12.6 .1 .2 .3])$. As the central character of $g_{0}$ is trivial, $\eta_{v_{0}}^{\prime}=\mu^{-2}$, hence $\eta_{v_{0}}^{\prime}(-1)=1$. This would imply that $1=\prod_{w_{0}} \eta_{w_{0}}^{\prime}(-1)=(-1)^{\left[F_{0}: \mathbb{Q}\right]}$, which contradicts our assumption.

3.3.3. - Let us now prove the last line in the statement of Theorem 0.8(2). For each $s=$ $0, \ldots, r, \rho_{s}=\operatorname{Ind}_{K_{s} / F_{0}}\left(\chi_{s}\right)$ is an absolutely irreducible $L\left[G_{F_{0}}\right]$-module of dimension $\varphi\left(q^{s}\right)$. If $1 \leq s \leq r$, then $\rho_{s} \otimes_{L} L_{\mathfrak{p}}$ occurs in $H_{f}^{1}\left(K_{r}(\sqrt[q r]{a}), V\right)$ with odd multiplicity, by Shapiro's Lemma combined with Theorem 0.4(1) applied to each $g_{s}$ and $\chi_{s}$. As the trivial representation $\rho_{0}$ occurs with multiplicity $h_{f}^{1}\left(F_{0}, V\right)$, it follows that

$$
h_{f}^{1}\left(K_{r}\left(\sqrt[q^{r}]{a}\right), V\right)-h_{f}^{1}\left(F_{0}, V\right) \geq \sum_{s=1}^{r} \operatorname{dim}\left(\rho_{s}\right)=\sum_{s=1}^{r} \varphi\left(q^{s}\right)=q^{r}-1 .
$$

\section{REFERENCES}

[1] E. Aflalo, J. Nekovář , Non-triviality of CM points in ring class field towers, to appear in Israel J. Math. .

[2] S. Bloch, K. Kato, L-functions and Tamagawa numbers of motives, in The Grothendieck Festschrift, Vol. I, Progr. Math. 86, Birkhäuser, 1990, 333-400.

[3] J. Coates, T. Fukaya, K. Kato, R. Sujatha, Root numbers, Selmer groups and noncommutative Iwasawa theory, preprint.

[4] C. Cornut, V. Vatsal, Nontriviality of Rankin-Selberg $L$-functions and CM points, in L-functions and Galois representations (Durham, July 2004), LMS Lecture Note Series 320, Cambridge Univ. Press, 2007, 121-186.

[5] T. Dokchitser, V. Dockchitser, Regulator constants and the parity conjecture, preprint arXiv:0709.2852. 
[6] T. Dokchitser, V. Dokchitser, On the Birch-Swinnerton-Dyer quotients modulo squares, preprint arXiv:math/0610290.

[7] V. Dokchitser, Root numbers of non-abelian twists of elliptic curves, Proc. London Math. Soc. 91 (2005), 300-324.

[8] J.-M. Fontaine, B. Perrin-Riou, Autour des conjectures de Bloch et Kato: cohomologie galoisienne et valeurs de fonctions $L$, in Motives (Seattle, WA, 1991), Proc. Sympos. Pure Math. 55, Amer. Math. Soc., 1994, 599-706.

[9] B. H. Gross, D. Prasad, Test vectors for linear forms, Math. Ann. 291 (1991), 343355.

[10] H. Jacquet, Automorphic forms on GL(2). Part II, Lecture Notes in Math. 278, Springer, 1972.

[11] H. Jacquet, R. P. Langlands, Automorphic forms on GL(2), Lecture Notes in Math. 114, Springer, 1970.

[12] B. Mazur, K. Rubin, Finding large Selmer rank via an arithmetic theory of local constants, Ann. of Math. 166 (2007), 579-612.

[13] J. Nekovář, Selmer complexes, Astérisque 310 (2006), 559.

[14] J. Nekovář, On the parity of ranks of Selmer groups. III, Doc. Math. 12 (2007), 243274.

[15] J. Nekovář, The Euler system method for CM points on Shimura curves, in $L$ functions and Galois representations (Durham, July 2004), LMS Lecture Note Series 320, Cambridge Univ. Press, 2007, 471-547.

[16] J. Nekovář, On the parity of ranks of Selmer groups IV, to appear in Compositio Math.

[17] H. Saito, On Tunnell's formula for characters of GL(2), Compositio Math. 85 (1993), 99-108.

[18] J. B. Tunnell, Local $\epsilon$-factors and characters of GL(2), Amer. J. Math. 105 (1983), 1277-1307.

[19] J.-L. Waldspurger, Correspondances de Shimura et quaternions, Forum Math. 3 (1991), 219-307.

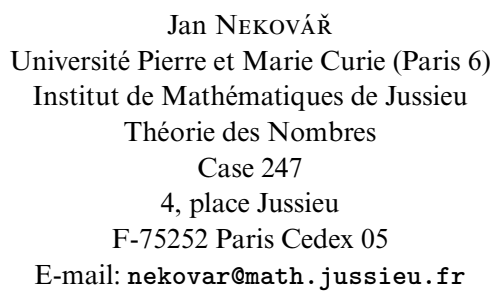

